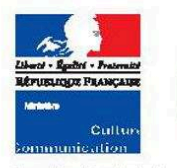

Secrétariat général

Service de la

politiques culturelles

et de l'innovation

Département

des études,

de la prospective
et des statistiques

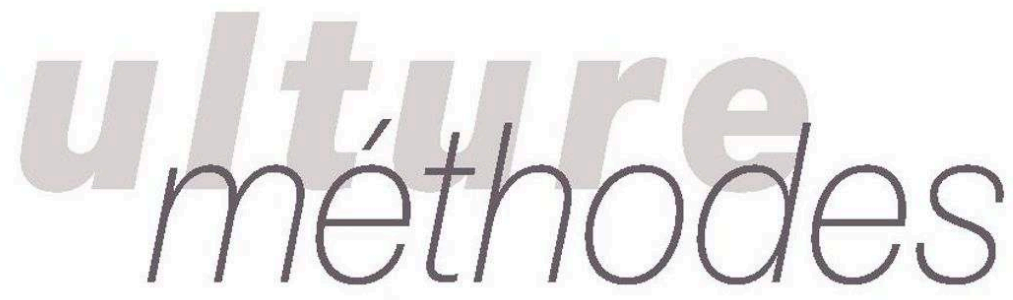

82, rue Saint-Honoré, 75033 Paris cedex 0

중 0140157917 -国 0140157999

\title{
Une mesure de la diversité des marchés du film en salles et en vidéogrammes en France et en Europe
}

\section{$D^{r}$ Florence Lévy-Hartmann*}

An Evaluation of the Diversity of the Film Market for Cinema Avant-propos and Video Recordings in France and in Europe

La diversité culturelle est aujourd' hui un objectif central des politiques publiques culturelles, en particulier depuis l'adoption de la Convention sur la protection et la promotion de diversité des expressions culturelles de l'Unesco en 2005, en vigueur depuis te 18 mars 2007 après sa ratification par la France et l'Union européenne. De nombreuses mesures de politique culturelle s'y réfêrent, notamment dans les secteurs des industries culturelles. Préoccupation principalement potitique, la diversité culturelle n'en possède pas moins des dimensions économiques car elle renvoie à des questions importantes - concurrence, concentration industrielle, pouvoir de marché et optimalité économique - qui se trouvent par ailleurs, dans le contexte actuet, renowelées par une globalisation économique et les effets produits par la révolution numérique.

Conscient des enjeux de politique publique que pose la question de la diversité, le DEPS l'a inscrit, depuis plusieurs années, dans son programme de recherche'. En 2008, il a lancé un appet a propositions de recherche sur "l appréciation et les processus de modification de la diversite dans les filleres d industrie culturelle *. Visant à enrichir t'ensemble des résultats de l'analyse socioéconomique sur le sujet, lappet invilat les thercheurs à apporter des elements de reponse quant à mesures! et l'identification des processus par lesquels la diversité des produits mesures, et lidentification des processus par lesquets la
culturets offerts, distribués et consommés a pu se modifier.

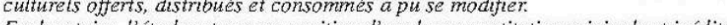
Exploratoire, leude est une proposition d analyse quantitative originale et inédite

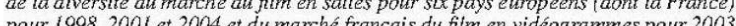

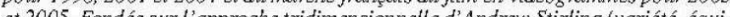

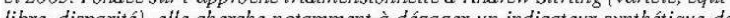
libre, disparite, elle cherche notamment à degager un indicateur synthétique de diversité puisque par comer lexcellence de la polingue finçaise en maleve de 列 en salles.

Depuis une dizaine d'années, la problématique, la revendication, puis l'impératif de la diversité culturelle ${ }^{2}$ ont émergé dans les normes juridiques françaises, européennes et internationales. Qu'est-ce que la diversité culturelle d'un marché, quelle rationalité économique et sociale est à l'œuvre derrière l'exigence de diversité culturelle? Ces questions appellent la mise en place d'un cadre conceptuel strict afin de définir si la notion de diversité culturelle, essentiellement brandie comme argument politique, permet de fonder un discours économique.

Analysée sous l'angle de la mesure de la diversité des marchés culturels, qui nécessite que des méthodologies nouvelles soient élaborées, la notion de diversité culturelle s'applique à tous les marchés culturels, en particulier au secteur cinématographique, du fait de son importance symbolique et historique et de sa place à part dans les négociations commerciales internationales ${ }^{3}$.

* Laboratoire Groupe d'économie mondiale (GEM), Sciences Po.

1. Voir notamment Tristan MATTELART, Enjeux intellectuels de la diversité culturelle. Eléments de déconstruction théorique, Paris, Ministère de la Culture et de la Communication, DEPS, coll. « Culture prospective », 2009-2, juillet 2009. Voir aussi Hélène HATZFELD, Vincent BILLEREY, Repères pour un dialogue interculturel, Ministère de la Culture et de la Communication, Secrétariat général (SCFCJ/DREST), 2010, en ligne sur www.culture.gouv.frírubr que Politiques ministerielles.

2. La Déclaration universelle sur la diversité culturelle adoptée par les membres de l'Unesco en 2001 puis la Convention sur la protection et la promotion de la diversité des expressions culturelles en 2005; les articles 87 et 151 du traité instituant la Communauté européenne (TCE), la Charte des droits fondamentaux et le protocole no 9 annexé au traité d'Amsterdam (1997), puis l'article 167 du traité sur le fonctionnement de l'Union européenne (TFUE) ; enfin la résolution du Conseil européen du 25 janvier 1999 concernant le service public de radiodiffusion, la résolution du Conseil européen sur les aides nationales au cinéma et à l'audiovisuel du 12 février 2001, le programme Media 2007 (2007-2013), la directive européenne «services de médias audiovisuels 》 (SMAV) du 11 décembre 2007 ou encore la stratégie « agenda numérique pour l'Europe » (2010-2020).

3. Les conflits commerciaux relatifs aux films sont nés avec le cinéma lui-même : les premiers conflits commerciaux relatifs à la circulation des films remontent aux années 1910 et voient déjà s'opposer les entreprises cinématographiques européennes aux firmes américaines. L'une des dernières et des plus virulentes disputes s'est produite lors du cycle de négociation de l'Uruguay (1986-1994), dans le cadre des accords Gatt, c'est-à-dire des négociations commerciales multilatérales.

Directeur de publication: Jean-François Chaintreau, chef du service de la coordination des politiques culturelles

et de l'innovation, en charge du département des études, de la prospective et des statistiques.

$2011-1$ - octobre 2011

Responsable des publications: Edwige Millery 


\title{
Une mesure de la diversité des marchés du film en salles et en vidéogrammes en France et en Europe
}

\author{
An Evaluation of the Diversity of the Film Market for Cinema and Video Recordings in France and in Europe
}

Florence Lévy-Hartmann

Éditeur : Département des études, de la prospective et des statistiques

Lieu d'édition : Paris

Année d'édition : 2011

Date de mise en ligne : 21 septembre 2015

Collection : Culture méthodes

ISBN électronique : 9782111397972

\section{Sobooks}

http://books.openedition.org

\section{Édition imprimée}

Date de publication : 30 octobre 2011

Nombre de pages : 16

\section{Référence électronique}

LÉVY-HARTMANN, Florence. Une mesure de la diversité des marchés du film en salles et en

vidéogrammes en France et en Europe. Nouvelle édition [en ligne]. Paris : Département des études, de la prospective et des statistiques, 2011 (généré le 25 avril 2021). Disponible sur Internet : <http://

books.openedition.org/deps/327>. ISBN : 9782111397972. 

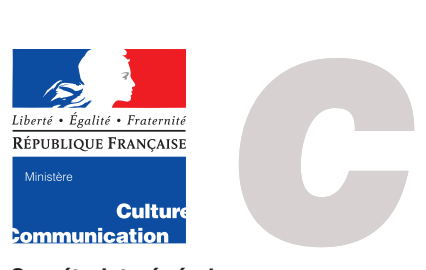

Secrétariat général

Service de la

coordination des

politiques culturelles

et de l'innovation

Département

des études,

de la prospective

et des statistiques

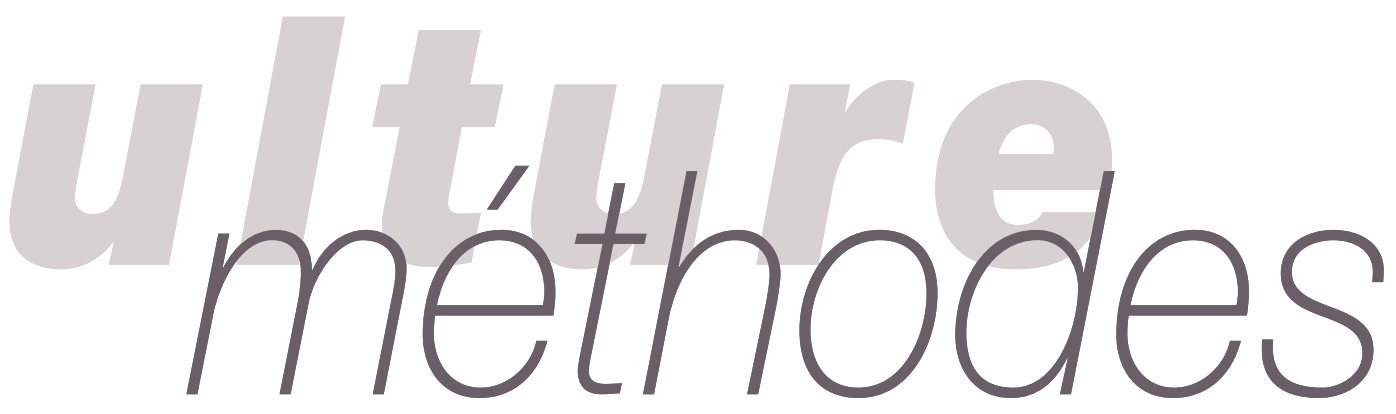

182, rue Saint-Honoré, 75033 Paris cedex 01

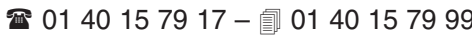

ÉCONOMIE DE LA CULTURE ET DE LA COMMUNICATION

2011-1

\section{Une mesure de la diversité des marchés du film en salles et en vidéogrammes en France et en Europe}

\section{$D^{r}$ Florence Lévy-Hartmann* \\ An Evaluation of the Diversity of the Film Market for Cinema Avant-propos and Video Recordings in France and in Europe}

La diversité culturelle est aujourd'hui un objectif central des politiques publiques culturelles, en particulier depuis l'adoption de la Convention sur la protection et la promotion de diversité des expressions culturelles de l'Unesco en 2005, en vigueur depuis le 18 mars 2007 après sa ratification par la France et l'Union européenne. De nombreuses mesures de politique culturelle s'y réfèrent, notamment dans les secteurs des industries culturelles. Préoccupation principalement politique, la diversité culturelle n'en possède pas moins des dimensions économiques car elle renvoie à des questions importantes - concurrence, concentration industrielle, pouvoir de marché et optimalité économique - qui se trouvent par ailleurs, dans le contexte actuel, renouvelées par une globalisation économique et les effets produits par la révolution numérique.

Conscient des enjeux de politique publique que pose la question de la diversité, le DEPS l'a inscrit, depuis plusieurs années, dans son programme de recherche ${ }^{1}$. En 2008, il a lancé un appel à propositions de recherche sur "l'appréciation et les processus de modification de la diversité dans les filières d'industrie culturelle ». Visant à enrichir l'ensemble des résultats de l'analyse socioéconomique sur le sujet, l'appel invitait les chercheurs à apporter des éléments de réponse quant à l'appréciation des différentes dimensions de la diversité culturelle (définitions et mesures) et l'identification des processus par lesquels la diversité des produits culturels offerts, distribués et consommés a pu se modifier.

Exploratoire, l'étude est une proposition d'analyse quantitative originale et inédite de la diversité du marché du film en salles pour six pays européens (dont la France) pour 1998, 2001 et 2004 et du marché français du film en vidéogrammes pour 2003 et 2005. Fondée sur l'approche tridimensionnelle d'Andrew Stirling (variété, équilibre, disparité), elle cherche notamment à dégager un indicateur synthétique de diversité et tend à confirmer l'excellence de la politique française en matière de diversité puisque, par comparaison avec d'autres marchés européens, le marché français est le plus varié et le plus équilibré, notamment en termes d'offres de films en salles.

Jean-François ChaInTREAU
Depuis une dizaine d'années, la problématique, la revendication, puis l'impératif de la diversité culturelle ${ }^{2}$ ont émergé dans les normes juridiques françaises, européennes et internationales. Qu'est-ce que la diversité culturelle d'un marché, quelle rationalité économique et sociale est à l'œuvre derrière l'exigence de diversité culturelle ? Ces questions appellent la mise en place d'un cadre conceptuel strict afin de définir si la notion de diversité culturelle, essentiellement brandie comme argument politique, permet de fonder un discours économique.

Analysée sous l'angle de la mesure de la diversité des marchés culturels, qui nécessite que des méthodologies nouvelles soient élaborées, la notion de diversité culturelle s'applique à tous les marchés culturels, en particulier au secteur cinématographique, du fait de son importance symbolique et historique et de sa place à part dans les négociations commerciales internationales ${ }^{3}$.

* Laboratoire Groupe d'économie mondiale (GEM), Sciences Po.

1. Voir notamment Tristan Mattelart, Enjeux intellectuels de la diversité culturelle. Éléments de déconstruction théorique, Paris, Ministère de la Culture et de la Communication, DEPS, coll. « Culture prospective », 2009-2, juillet 2009. Voir aussi Hélène HATZFELD, Vincent BILLEREY, Repères pour un dialogue interculturel, Ministère de la Culture et de la Communication, Secrétariat général (SCPCI/DREST), 2010, en ligne sur www.culture.gouv.fr/rubri que Politiques ministérielles.

2. La Déclaration universelle sur la diversité culturelle adoptée par les membres de l'Unesco en 2001 puis la Convention sur la protection et la promotion de la diversité des expressions culturelles en 2005 ; les articles 87 et 151 du traité instituant la Communauté européenne (TCE), la Charte des droits fondamentaux et le protocole $\mathrm{n}^{\circ} 9$ annexé au traité d'Amsterdam (1997), puis l'article 167 du traité sur le fonctionnement de l'Union européenne (TFUE) ; enfin la résolution du Conseil européen du 25 janvier 1999 concernant le service public de radiodiffusion, la résolution du Conseil européen sur les aides nationales au cinéma et à l'audiovisuel du 12 février 2001, le programme Media 2007 (2007-2013), la directive européenne «services de médias audiovisuels » (SMAV) du 11 décembre 2007 ou encore la stratégie «agenda numérique pour l’Europe » (2010-2020).

3. Les conflits commerciaux relatifs aux films sont nés avec le cinéma lui-même : les premiers conflits commerciaux relatifs à la circulation des films remontent aux années 1910 et voient déjà s'opposer les entreprises cinématographiques européennes aux firmes américaines. L'une des dernières et des plus virulentes disputes s'est produite lors du cycle de négociation de l'Uruguay (1986-1994), dans le cadre des accords Gatt, c'est-à-dire des négociations commerciales multilatérales. 


\section{QU'EST-CE QUE LA DIVERSITÉ CULTURELLE?}

Omniprésente depuis le début des années 2000, l'idée de diversité culturelle se caractérise par une certaine confusion sémantique, la dilution du terme représentant sans doute, pour ses adversaires comme pour ses partisans, le moyen d'éluder la question de la définition de la notion.

La culture est définie par la déclaration de Mexico sur les politiques culturelles de 1982, réaffirmée à Stockholm en 1998, comme «l'ensemble des traits distinctifs, spirituels et matériels, intellectuels et affectifs qui caractérisent une société ou un groupe social ${ }^{4} »$. Ainsi se déploie l'ampleur du champ culturel et s'affirme la nécessité de restreindre le champ de la culture pour la rendre compatible avec l'idée de marché.

Pour pouvoir traiter des industries culturelles et du champ artistique, l'Unesco s'est volontairement limitée au champ des «contenus culturels et expressions artistiques », renommé depuis 2004 «expressions culturelles ». Ainsi la Convention adoptée en 2005 ne porte-t-elle pas, dans son intitulé, sur la diversité culturelle, mais bien sur la protection et la promotion de la diversité des expressions culturelles. La réduction du champ de la convention de la culture aux expressions culturelles permet de construire un texte juridique adapté à la réglementation internationale des politiques culturelles, ce qui aurait été impossible à partir de la définition large de la culture: «Aux fins de cette Convention, les politiques et mesures culturelles sont définies comme [...] politiques et mesures relatives à la culture, à un niveau local, national, régional ou international, qu'elles soient centrées sur la culture en tant que telle, ou destinées à avoir un effet direct sur les expressions culturelles des individus, groupes ou sociétés, y compris sur la création, la production, la diffusion et la distribution d'activités, de biens et de services culturels et sur l'accès à ceux-ci » (article 4.6).

Le marché cinématographique, considéré comme une des nombreuses «expressions culturelles », est envisagé dans ses trois dimensions : l'offre cinématographique, la distribution cinématographique, la demande cinématographique, synonyme ici de volume des entrées.

La diversité est bien plus objectivable que la culture, et son usage est à la fois courant ${ }^{5}$ et pointu dans de nombreuses disciplines scientifiques. Parmi l'abondance de références, deux textes semblent incontournables: l'un de Simpson ${ }^{6}$ paru en 1949, l'autre de Weitzman ${ }^{7}$, paru en 1992.

Dans un bref article d'une seule page, Simpson présente un indice de mesure de la diversité, qu'il définit comme le degré de concentration d'un nombre fini d'individus dans différents groupes ou espèces. L'indice formulé tient compte du nombre de groupes - c'est la variété ou richesse - et de la plus ou moins égale répartition des individus dans les différents groupes - c'est l'équilibre.

Weitzman insiste sur l'importance des coefficients de distance dans la définition de la diversité. Il propose une mesure de la distance entre les éléments d'un groupe : c'est la disparité. Mais sa fonction de diversité s'avère particulièrement contraignante. D'une part, elle repose uniquement sur la distance entre les éléments et ne tient pas compte de l'équilibre ou de la richesse du groupe. D'autre part, elle s'appuie sur une conception très précise, et finalement limitative, de la distance entre les éléments, qui impose que le groupe étudié se caractérise par une structure taxinomique.

Il est aujourd'hui largement admis que la diversité se compose des trois dimensions présentes dans les textes de Simpson et Weitzman : la variété, l'équilibre et la disparité ${ }^{8}$.

La diversité culturelle est donc envisagée comme la diversité des marchés de produits culturels. Celle-ci repose sur la variété, l'équilibre et la distance entre les produits offerts, distribués et consommés. Sur le fondement de cette définition, la mise en place d'un outil de mesure de la diversité des marchés cinématographiques devient possible. Plusieurs études se sont déjà penchées sur la question de la mesure de la diversité des marchés culturels, mais aucune méthodologie ne s'est encore imposée. Les études de cas doivent donc être multipliées afin de favoriser l'émergence d'une ou plusieurs méthodologies plus ou moins consensuelles.

\section{LA DIVERSITÉ DU MARCHÉ DU FILM EN SALLES EN EUROPE}

L'industrie du film de cinéma est composée d'une multitude de marchés: salles, vidéo (location, ventes, téléchargement), diffusion TV (premium, payante, gratuite), etc. Le choix, parmi les nombreux marchés du film et à l'heure du numérique et de la convergence, du marché traditionnel du film en salles s'explique d'abord par la puissance, symbolique mais également économique, de l'exploitation des films en salles dans la carrière des films ${ }^{9}$, et par la relative transparence du marché du film en salles : la disponibilité et une relative fiabilité des données de production, de fréquentation et dans une moindre mesure de distribution des films autorisent une étude intertemporelle et internationale des marchés du film dans les salles européennes.

\footnotetext{
4. D’une manière identique, la Déclaration universelle de 2001 sur la diversité culturelle (Paris, Unesco, 2001) présente une vision étendue de la culture comme «l'originalité et la pluralité des identités qui caractérisent les groupes et les sociétés qui composent l'humanité ».

5. Par exemple le Petit Robert spécifie « caractère, état de ce qui est divers ». Il fournit deux listes de mots ayant de grands rapports de sens avec la diversité, d'abord «multiplicité, pluralité, variété » puis « hétérogénéité, richesse ».

6. Edward H.Simpson, "Measurement of Diversity", Nature, avril 1949, vol. 163, p. 688.

7. Martin L.Weitzman, "On Diversity”, The Quarterly Journal of Economics, mai 1992, vol. 107, no 2, p 363-405.

8. Voir par exemple OECD, Handbook of Biodiversity Valuation: A Guide for Policy-Makers, Paris, OECD, 2002,156 p.

9. Le film en salles demeure le premier marché du film dans la chronologie des médias. Si la part des ventes de billets dans les recettes totales générées par les films a régulièrement diminué, les salles continuent d'attirer les spectateurs et la sortie sur grand écran constitue toujours un moment clé dans la carrière d'un film. En 2009, les salles européennes ont attiré 985 millions de spectateurs, dont 200 millions en France (Observatoire européen de l'audiovisuel).
} 


\section{Disponibilité et collecte des données}

L'analyse du marché du film en salles porte sur six marchés les plus représentatifs de la diversité des pays et marchés du film européens ${ }^{1}$ parmi les vingt-sept que compte l'Union européenne: Danemark, Espagne, France, Pologne, Royaume-Uni et Suède. L'étude est menée sur trois années: 1998, 2001 et 2004.

La première source de données utilisée est la base de données européenne LUMIERE, de l'Observatoire européen de l'audiovisuel. Créée en 2000, elle fournit une information détaillée sur la fréquentation des salles de cinéma en Europe ${ }^{2}$. LUMIERE propose à la fin de l'année 2010 des données pour plus de 20000 films, distribués dans une trentaine de pays, entre 1996 et 2009. Les films et chiffres de fréquentation compilés dans la base proviennent d'une multitude de sources nationales et européennes. Des données complémentaires à celles de LUMIERE sont recueillies via les différents instituts nationaux du film ${ }^{3}$, qui publient pratiquement tous des bases de données publiques sur l'internet, et différentes bases de données commerciales en ligne (IMDB, CBO, Box Office Mojo, etc.).

Une liste de 5650 films est constituée, qui compile l'intégralité des films exclusifs et les plus importants films de patrimoine (définis comme les films de plus de trois ans) dans les six pays au cours des trois années considérées (graphique 1).

Après la constitution de la liste de 5650 films, une quinzaine d'attributs est collectée dans un second temps sur chaque film de cette liste, dans l'objectif de les caractériser de la manière la plus détaillée possible et pertinente en termes de diversité. À cet égard sont recueillis : le nom du réalisateur, l'année de production, la nationalité, la langue, le rang, le budget de production, la qualité, la suite, la présence de stars, le producteur, le distributeur, l'éditeur ou encore le nombre de copies. Cette collecte détaillée constitue un préalable indispensable à l'élaboration d'une série de mesures de la diversité des marchés du film en salles (offre, distribution, demande 4 ).

L'échantillon de films sur les six marchés européens au cours des trois années choisies représente l'offre des films. Des données sont aussi collectées par rapport à leur distribution et à leur exploitation. Le nombre de copies en circulation, qui reflète la distribution, n'est disponible que pour deux des six pays (France et Pologne) et deux des trois années (2001 et 2004), mais le nombre d'entrées réalisées par chacun des films est recueilli pour l'intégralité de l'échantillon par l'intermédiaire de la base LUMIERE.

1. Il faut noter que les pays de l'échantillon présentent de significatifs écarts de taille et de population, des langues différentes (« grandes » et « petites » langues européennes), des situations géographiques variées (Europe de l'Ouest, de l'Est, pays nordique ou du Sud, etc.). Au-delà, l'échantillon tient compte de la variété des structures, des marchés et des réglementations cinématographiques en Europe: grands et petits pays en termes de production de films, pays plus ou moins influencés en termes commerciaux par le cinéma nord-américain et enfin pays caractérisés par des réglementations cinématographiques plus ou moins volontaristes.

2. Voir : http://lumiere.obs.coe.int/web/sources/histo.html

3. DFI au Danemark, ICAA en Espagne, CNC en France, Film Council au Royaume-Uni, Film Polski en Pologne et SFI en Suède.

4. Elle permet aussi de dresser un panorama de la disponibilité des données relatives aux films de cinéma en Europe et de forger une batterie de statistiques

élémentaires parfois inédites sur les marchés européens du film. Pour plus de détails, voir Florence LEVY-HARTMANN, la Diversité culturelle des marchés du film en salles et du film en vidéogrammes en France et en Europe, document de travail, Paris, ministère de la Culture et de la Communication, 2011.

\section{Répartition des films en catégories}

La diversité se compose de trois dimensions (richesse, équilibre et disparité), mais rares sont les indices proposant leur combinaison synthétique : les indicateurs n'envisagent généralement que l'une ou l'autre, voire deux des trois dimensions. En particulier, l'appréhension de la disparité pose problème : comment identifier et mesurer la distance entre deux films?
Il convient, au préalable, d'établir une nomenclature de catégories de films. Les films d'une même catégorie sont relativement similaires entre eux, tandis que les films appartenant à des catégories éloignées sont naturellement marqués par une grande dissimilarité.

Il s'agit ici de défendre l'idée d'un certain degré de concurrence monopolistique sur le marché du film, d'un degré non négligeable de substituabilité entre les films. Bien que chaque film soit une œuvre unique et que

\section{Graphique 1 - Composition de la liste des 5650 films par pays et par année}

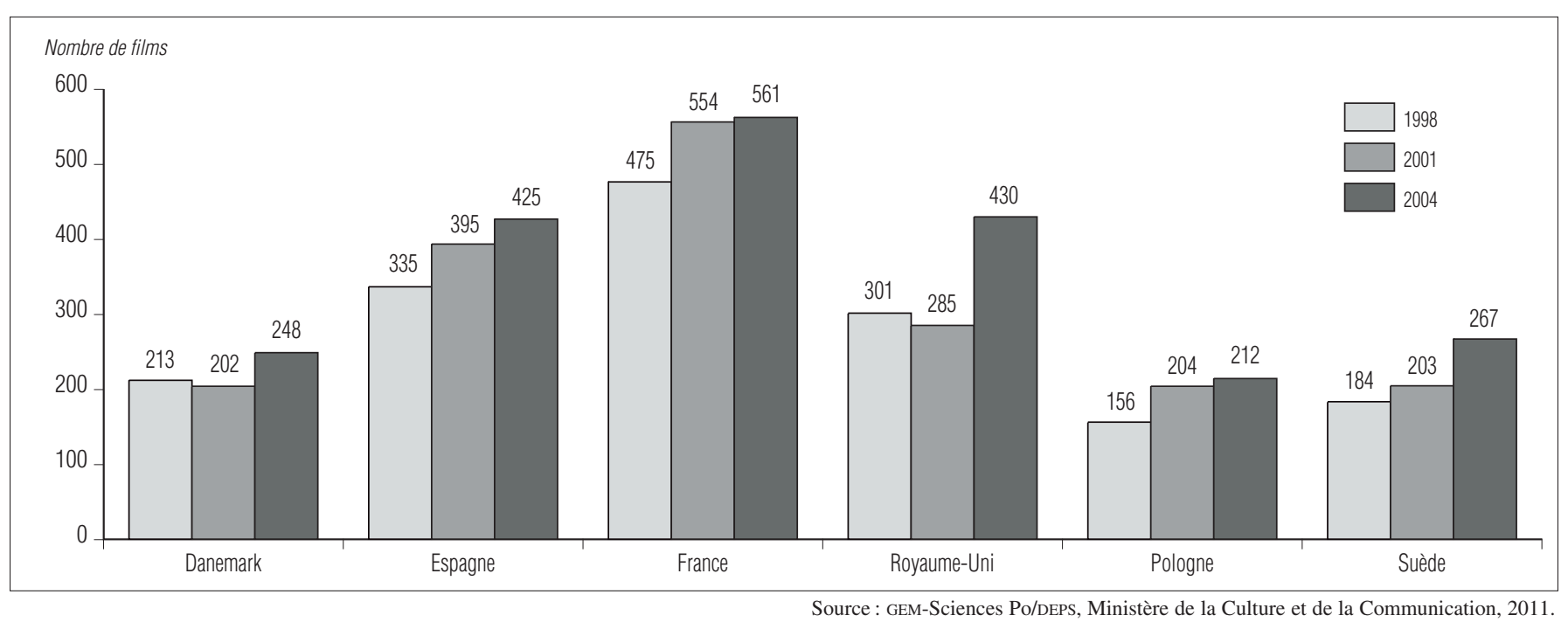


l'appréciation de chaque spectateur demeure subjective, il semble envisageable d'un point de vue scientifique d'établir des rapprochements entre des types de films.

La création de catégories de films permet non seulement d'appréhender la variété et l'équilibre entre les films, mais aussi la disparité entre eux. C'est donc bien la création d'une nomenclature qui permet d'envisager la mesure de chacune des trois dimensions de la diversité et la mise en œuvre d'un indicateur synthétique de diversité.

La nomenclature est articulée autour de plusieurs critères, pour éviter d'étudier la diversité à partir d'un unique indicateur, trop élémentaire (en général la nationalité des films).

\section{Attributs retenus pour la création d'une nomenclature de films}

Plusieurs attributs doivent être croisés afin de générer une nomenclature respectueuse de la complexité de l'objet «film»; cependant, le nombre de caractéristiques ne doit pas non plus être trop important, sous peine de ne plus pouvoir regrouper les films en catégories significatives et opérationnelles et d'obtenir une nomenclature illisible. Enfin, le choix des attributs tient aussi compte d'un principe de réalité qui conduit à écarter ceux qui n'ont pu être parfaitement collectés (ainsi les budgets de production et de promotion des films).

La nomenclature établie fait écho aux trois dimensions de la diversité culturelle dégagées par Paris en $2003^{10}$ : géographique, industrielle et artistique. En effet, elle combine des informations relatives à l'ancrage culturel, défini comme la combinaison de la nationalité et de la langue des films (la diversité géographique), les structures écono- miques (la diversité industrielle) et l'exigence artistique/la qualité ${ }^{11}$ ainsi que l'âge des films (la diversité artistique).

Les cinq critères retenus pour la construction de la nomenclature sont généralement indépendants des conditions de distribution puis de l'accueil réservé par le public au film : l'ancrage culturel du film et la structure de production sont déterminés avant la mise sur le marché du film ; l'indice de qualité est construit à partir de l'attribution (ou non) de la recommandation «Art et Essai » ou des critiques reçues par le film (celles-ci sont attribuées ou publiées juste avant la sortie d'un film sur un marché).

\section{Une nomenclature de films : dix-neuf catégories de films}

La combinaison des critères définis ci-dessus permet d'élaborer une nomenclature de dix-neuf catégories de films combinant l'ancrage culturel (nationalité et langue), la structure de production, la qualité et l'âge du film.

Afin d'illustrer la nomenclature, le tableau présente, pour chacune des catégories, un titre de film l'incarnant, l'illustrant plus concrètement que le seul code ${ }^{12}$ (tableau 1). Dix-neuf titres de films figurent donc dans la nomenclature à titre d'exemple, chacun étant bien sûr issu de la catégorie $^{13}$ qu'il représente.

Les 5650 films sont donc répartis dans les dix-neuf catégories de films à partir desquelles des tableaux d'effectifs et de fréquences peuvent être établis. Ces tables servent de support aux analyses des trois dimensions puis à l'analyse synthétique de la diversité des marchés, relativement à l'offre, à la distribution et à la demande de films dans six pays européens au cours de trois années.

\section{Des catégories de films?}

Regrouper les films, objets uniques différenciés, dans des sous-ensembles relativement homogènes, c'est-à-dire dans des " espèces ", artificielles et multidimensionnelles, peut choquer. II s'agit pourtant, selon nous, d'un préalable essentiel à la constitution d'un indicateur de la diversité, à la formation d'un discours rationnel sur la diversité des marchés du cinéma. II s'agit surtout de sortir de la traditionnelle alternative :

- analyser la diversité à partir d'un indicateur unidimensionnel, construit autour d'un attribut unique (souvent la nationalité des films). Le discours est alors réducteur, il est certes simple et efficace mais ne porte en réalité pas sur la diversité des marchés;

- analyser la diversité à partir d'un faisceau d'indicateurs hétérogènes, qui peuvent être analysés un par un, et les conclusions de ces analyses juxtaposées. L'analyse est alors multidimensionnelle, et en ce sens plus proche de ce qu'est réellement la diversité des marchés du film. Mais comment tirer des enseignements depuis une batterie d'indicateurs disparates ? L'analyse ne peut être ni synthétique, ni par conséquent conclusive.

La construction d'une nomenclature de films multidimensionnelle permet de résoudre ce dilemme en autorisant une analyse à la fois opérationnelle (i.e. faisable et significative d'un point de vue statistique) et pluridimensionnelle, donc pas trop réductrice (i.e. les films ne sont pas réduits à un attribut unique).

10. Voir Maryvonne de Saint Pulgent, Pierre-Jean Benghozi, Thomas Paris, Mondialisation et diversité culturelle : le cas de la France, Paris, Les notes de l'Ifri, 2003, 84 p.

11. Le choix d'introduire le critère d'exigence artistique ou de qualité dans la nomenclature est fort : il s'agit de ne pas occulter la dimension artistique de l'analyse de la diversité des marchés du film, car, après tout, c'est bien de la dimension culturelle et artistique des marchés du film que naît l'impératif de protéger et de promouvoir leur diversité. L'attribut est renseigné par le label Art et Essai obtenu ou non par les films, qui ont connu une sortie dans les salles françaises, et par l'accueil critique pour les autres (accueil positif ou négatif tel que compilé sur des sites comme Rotten Tomatoes ou Metacritic).

12. Le code est constitué de la manière suivante : l'ancrage culturel du film détermine le premier chiffre (de 1 à 8 ), la structure de production ( 0 pour major et 1 pour indépendante) détermine le second chiffre pour les catégories concernées (les films américains en anglais, donc l'ancrage culturel 1). La qualité ( 0 pour faible et 1 pour élevée) détermine le dernier chiffre du code. Enfin, la catégorie 9 est réservée aux films de patrimoine et/ou muets. 13. Lorsque les catégories sont fondées sur des attributs comme le pays et la langue domestique, les exemples donnés sont tirés du marché français. Bien sûr, Vidocq ou L'Anglaise et le duc ne figurent pas dans les catégories «30 » et «31 » pour l'analyse des marchés britannique ou espagnol. 


\section{Variété, équilibre et disparité du marché du film en salles}

\section{Variété du marché du film en salles}

La variété, ou richesse, représente l'étendue du choix offert, concrètement distribué et finalement consommé. Plus le nombre d'options proposées ou choisies est élevé, plus la richesse sera considérée comme importante. Comment appréhender la richesse des films sur un marché en termes d'offre, de distribution et de demande?

Il s'agit de mesurer la variété d'une collection N, constituée d'entités $(n)$ regroupées en catégories $(\mathrm{S})$. Quatre indicateurs de variété sont utilisés :

- le nombre d'espèces $(\mathrm{S})$ présentes dans la collection (au niveau de l'offre, de la distribution puis de la demande). Indicateur traditionnel de variété, notamment utilisé dans le domaine de la biologie, il ne paraît pas toutefois complètement pertinent ${ }^{14}$;

- le nombre de produits $(\mathrm{N})$ présents dans la collection ${ }^{15}$ : nombre de films offerts/de copies en circulation/de billets vendus. L'outil présente l'avantage de n'être pas biaisé par la définition subjective des espèces de films mais il pose la question de la comparaison entre la variété offerte, celle distribuée et celle demandée ${ }^{16}$. Surtout, il demeure sensible à la taille du marché considéré. D'où, dans la perspective de comparaisons internationales, la nécessité de tester des indicateurs pondérés ;

- le nombre de produits (films/copies/billets) pondéré par la population (exprimée en milliers d'habitants) ;

- le nombre de produits (films/copies/billets) pondéré par le parc d'écrans (exprimé en centaines d'écrans).

L'analyse de ces quatre indicateurs permet de tirer certaines conclusions relatives à la richesse des six marchés de l'échantillon entre 1998 et 2004 :

- bien que les différents indicateurs établissent des hiérarchies mouvantes entre les pays, les variétés de la distribution, de la demande, et dans une moindre mesure de l'offre, tendent à être plus importantes sur les grands marchés audiovisuels (France, Espagne et Royaume-Uni) que sur les petits (Danemark, Pologne et Suède ${ }^{17}$ );

\section{Tableau 1 - Nomenclature des films}

\begin{tabular}{|c|c|c|c|c|}
\hline Code & Exemple de films & $\begin{array}{l}\text { Ancrage culturel de production } \\
\text { (nationalité + langue) }\end{array}$ & $\begin{array}{l}\text { Structure } \\
\text { de production }\end{array}$ & Qualité \\
\hline \multicolumn{5}{|c|}{ Films récents parlants } \\
\hline 100 & Catwoman & \multirow[t]{4}{*}{ États-Unis + anglais } & \multirow[t]{2}{*}{ Major } & Non \\
\hline 101 & Gatacca & & & Oui \\
\hline$\overline{110}$ & Psycho Beach Party & & \multirow[t]{2}{*}{ Indépendante } & Non \\
\hline 111 & Requiem for a Dream & & & Oui \\
\hline 20 & Cold Mountain & \multirow{2}{*}{\multicolumn{2}{|c|}{$\begin{array}{l}\text { Pays anglophone (hors États-Unis) } \\
\text { Pays domestique, grand pays européen + anglais }\end{array}$}} & Non \\
\hline 21 & Intimacy & & & Oui \\
\hline 30 & Vidocq & \multirow[t]{2}{*}{ Pays domestique + langue domestique } & & Non \\
\hline 31 & L'Anglaise et le duc & & & Oui \\
\hline 40 & Go for Gold! & \multirow{2}{*}{\multicolumn{2}{|c|}{$\begin{array}{l}\text { États-Unis, pays anglophone, grand pays européen } \\
+ \text { langue domestique }\end{array}$}} & Non \\
\hline 41 & Madame Brouette & & & Oui \\
\hline 50 & The Passion of the Christ & \multicolumn{2}{|l|}{ Pays domestique ou anglophone } & Non \\
\hline 51 & $S-21$ & \multicolumn{2}{|l|}{ + ni anglais ni langue domestique } & Oui \\
\hline 60 & Autobahnraser & & Non \\
\hline 61 & La mala educación & \multicolumn{2}{|l|}{$\begin{array}{l}\text { Ou petit pays européen + anglais, langue domestique, } \\
\text { grande langue européenne }\end{array}$} & Oui \\
\hline 70 & Final Fantasy & \multirow{2}{*}{\multicolumn{2}{|c|}{$\begin{array}{l}\text { Reste du monde* + anglais, langue domestique, } \\
\text { grande langue européenne }\end{array}$}} & Non \\
\hline 71 & Central do Brasil & & & Oui \\
\hline 80 & The Attack of the Giant Mousaka & \multirow{2}{*}{\multicolumn{2}{|c|}{$\begin{array}{l}\text { Europe ou reste du monde }+ \\
\text { petite langue européenne ou autre langue }\end{array}$}} & Non \\
\hline 81 & Saraband & & & Oui \\
\hline \multicolumn{5}{|c|}{ Films de plus de trois ans et/ou films muets } \\
\hline 9 & Les 400 coups & \multicolumn{3}{|l|}{ Films de patrimoine et films muets } \\
\hline
\end{tabular}

14. Il suffit d'un seul film, d'une seule copie ou d'un unique billet pour que soit comptabilisée une catégorie au titre de la variété sur le marché, ce qui peut sembler parfois trop grossier.

15. Cette démarche correspond à celle employée par Benhamou et Peltier dans leur étude de la diversité du marché du livre (F. BENHAMOU, S. PELTIER, «Une méthode multicritères d'évaluation de la diversité culturelle... », art. cité).

16. Comment comparer la richesse du nombre de films offerts (5 650), du nombre de copies (149 940) et du nombre de billets vendus (1 477 millions) ? 17. L'analyse en nombre de catégories montre une richesse supérieure en Espagne (offre et demande) et en France (offre, distribution et demande) par rapport aux autres pays. Le Royaume-Uni suit, en sachant que deux catégories sont mécaniquement absentes sur ce marché. Le nombre de produits sur le marché (films, copies ou billets) renforce nettement le caractère plus varié des trois grands marchés par rapport aux petits pays. Les pondérations de la variété des films/des copies/des billets par la population ou le nombre d'écrans ne modifient finalement cette conclusion que dans le cas de l'offre, le Danemark et la Suède présentant alors nettement plus de films par écrans et par habitants que les trois grands pays. 
- l'analyse temporelle est cohérente entre les indicateurs et d'un échelon de la filière à l'autre : les variétés offertes, distribuées et consommées augmentent entre 1998 et $2004^{18}$;

- les niveaux de variété s'avèrent difficilement comparables entre les échelons de la filière (offre, distribution, demande). Quels que soient les indicateurs choisis, il est malaisé de comparer les variétés offertes, distribuées et finalement consommées : soit elles sont très proches (en termes d'espèces), soit elles ne peuvent être placées sur le même plan et sont finalement trop éloignées (en termes de produits).

\section{Équilibre du marché du film en salles}

Le calcul de l'équilibre (ou balance) du marché du film en salles s'attache au poids ou à l'importance respective des différentes catégories de films dans l'offre, la distribution ou la demande. La littérature économique est conséquente sur les questions d'équilibre, de concentration sur un marché et de mesure de ces concentrations; l'enjeu réside donc dans le choix entre les nombreux indicateurs.

\section{Cinq indicateurs d'équilibre sont testés}

Parmi les outils existants relatifs à la mesure de l'équilibre d'un marché, certains mesurent strictement l'équilibre du marché (parfois de manière relativement rudimentaire comme avec les indicateurs de dominance ${ }^{19}$ ), tandis que d'autres appréhendent à la fois l'équilibre et la variété du marché :

- l'indice de Berger-Parker est un indice simple de dominance. Il est constitué par la fréquence de la catégorie la plus importante :

$$
\mathrm{E} 1=\operatorname{nmax} / \mathrm{N}
$$

- le coefficient de concentration est aussi un indice de dominance. Il est formé ici par la somme des fréquences (pi) des trois plus importantes catégories :

$$
\mathrm{E} 2=\mathrm{pi}+\mathrm{pj}+\mathrm{pk}
$$

- l'indice d'Herfindahl-Hirschman est la somme des carrés des fréquences, c'est un indicateur très commun de mesure de la concentration sur un marché :

$$
\mathrm{E}_{3}=\Sigma \mathrm{p}_{\mathrm{i}}^{2}
$$

Un indicateur HH élevé (> 2000) signifie une forte concentration du marché ;

- l'indice d'équilibre de McIntosh est un indicateur de l'équilibre, et seulement de l'équilibre, entre différentes catégories au sein d'une collection, qui repose sur les effectifs dans chaque catégorie.

$$
\left.\mathrm{E}_{4}=\left[\mathrm{N}-\sqrt{\left(\Sigma \mathrm{n}_{\mathrm{i}}^{2}\right)}\right] / \mathrm{N}-(\mathrm{N} / \mathrm{N} \sqrt{\mathrm{S}})\right]
$$

Un nombre plus petit - ou une valeur absolue du résultat plus grande - est plus souhaitable du point de vue de l'équilibre ;

- l'indice dual (dit de diversité) de McIntosh prend en compte à la fois l'équilibre entre les catégories et la variété, à travers le nombre d'espèces représentées dans la collec$\operatorname{tion}^{20}$ :

$$
\mathrm{E}_{5}=\left[\left(\mathrm{N}-\sqrt{\left(\Sigma \mathrm{n}_{\mathrm{i}}^{2}\right)}\right] /[\mathrm{N}-\sqrt{\mathrm{N}}]\right.
$$

Il s'agit de la forme normalisée de la formule de diversité de McIntosh, si bien que les résultats varient entre 0 et 1 . Un résultat plus élevé signifie une diversité plus importante dans la collection.

Les cinq indicateurs permettent de dégager une série de résultats sur l'équilibre des marchés du film en salles :

- le marché français se révèle sensiblement plus équilibré que les autres. Les trois grands marchés, en tête desquels se trouve la France, présentent d'ailleurs des offres plus équilibrées que les petits pays. L'équilibre des copies est lui aussi le plus important en France. Enfin, l'équilibre de la demande est plus marqué en France, puis dans les trois petits pays, mais moins élevé en Espagne et au Royaume-Uni, où la fréquentation semble particulièrement concentrée sur quelques catégories de films ;

- les équilibres semblent en hausse en Europe par rapport à 1998. Sans pouvoir parler de hausse tendancielle, 1998, l'année de la sortie du Titanic de James Cameron, apparaît comme une très mauvaise année en termes d'équilibre $^{21}$;

- l'équilibre se dégrade de l'offre jusqu'à la demande. Contrairement à la mesure de la variété, la comparaison entre les équilibres des trois marchés (offre, distribution, demande) fait sens. Et le constat est sans appel : l'équilibre des billets vendus est sensiblement moindre par rapport à celui des copies, qui est lui-même nettement plus réduit que celui des films offerts sur le marché22.

\footnotetext{
18. La fréquentation par écran progresse particulièrement entre 1998 et 2001 (+3,2\%) puis à un rythme moins soutenu entre 2001 et 2004 . Le nombre de copies par écran augmente de plus de $16 \%$ entre les deux seules périodes considérées. Enfin, le nombre de films par centaines d'écrans diminue entre 1998 et 2001, mais progresse sensiblement au cours de la période suivante, pour finalement dépasser en 2004 le niveau de 1998.

19. Les indices de dominance (Berger-Parker et coefficient de concentration) sont certes des indicateurs légers et aisés à manipuler, mais leur pouvoir explicatif demeure limité. Ils n'envisagent pas, par définition, l'équilibre de toutes les catégories sur un marché mais seulement la part d'une ou de quelques catégories dominantes. Ils sont facilement influencés par quelques épiphénomènes sur les marchés nationaux et ne peuvent rendre compte de la représentation des petites catégories de films, comme les films des pays tiers ou les films de patrimoine, pourtant essentielles lorsque l'on s'intéresse à la diversité culturelle en matière cinématographique. S'il ne fallait retenir qu'un indicateur d'équilibre, mieux vaudrait alors éviter un indice de dominance.

20. De manière intéressante, on constate que l'indice dual de McIntosh mène à des conclusions toujours identiques à celles des deux indices d'équilibre pur. La prise en compte de la variété n'aurait alors aucun impact? Ceci s'explique sans doute par le fait que la variété considérée par cet indicateur est la variété en termes de catégories. Cette dernière s'est avérée peu discriminante entre les pays et les années. D'où les conséquences relativement marginales de sa prise en compte dans la formule duale de McIntosh.

21. L'équilibre de l'offre, plutôt élevé, semble progresser dans tous les pays entre 1998 et 2001 puis jusqu'en 2004. L'équilibre entre les copies (en France et en Pologne) régresse nettement entre 2001 et 2004. Dans tous les pays de l'échantillon, l'équilibre entre les billets vendus s'améliore nettement entre 1998 et 2001 puis se dégrade, de manière moins marquée, entre 2001 et 2004.

22. Par exemple, l'indice Herfindahl-Hirschman montre que l'offre globale se situe nettement sous le seuil de 2000 , mais que celui-ci est franchi lorsque l'on s'intéresse aux copies en France et en Pologne (2 381). Au niveau de la demande, l'indicateur s'établit à 3472 , ce qui montre une concentration marquée sur quelques catégories de films.
} 


\section{Disparité du marché du film en salles}

La disparité, ou distance, représente la dimension de la diversité la plus problématique d'un point de vue technique et la plus polémique d'un point de vue conceptuel. Elle soulève deux questions : peut-on mesurer la distance entre deux films et est-on en droit d'établir une telle mesure?

Différentes visions de la disparité entre deux catégories coexistent. Par exemple, notre nomenclature ne permet pas d'appliquer la distance ultramétrique entre les espèces développées par Weitzman. En revanche, une approche en termes de dissimilarité, qui offre l'avantage d'être multidimensionnelle, peut être mise en place. Il s'agit de comparer les espèces deux à deux, et attribut par attribut ${ }^{23}$. De là, une matrice de distance, ou plus exactement de dissimilarité, est élaborée ${ }^{24}$ :

À partir de la matrice de dissimilarité, deux calculs de la dissimilarité entre les films de l'échantillon sont proposés :

- la distance totale entre les catégories présentes dans l'échantillon, selon la méthodologie de Stirling ${ }^{25}$. Ce calcul répond à la stricte définition de la distance sur le marché, et ne tient pas compte du nombre de films, copies ou billets dans chaque catégorie ;

$$
\mathrm{D}_{1}=\Sigma \mathrm{d}_{\mathrm{ij}}
$$

où $\mathrm{d}_{\mathrm{ij}}$ est la distance entre les espèces $\mathrm{i}$ et $\mathrm{j}$.

- la distance totale entre les films de l'échantillon. À la différence de l'indicateur précédent, cette formule est très sensible au nombre de films proposés, à la richesse du marché. En prenant en compte les produits et non plus seulement la présence de catégories sur le marché, cette formule tient en effet aussi compte de la richesse sur le marché. Elle n'est donc pas indépendante des autres dimensions de la diversité :

$$
\mathrm{D}_{2}=\Sigma \mathrm{d}_{\mathrm{ij}} \mathrm{n}_{\mathrm{i}}
$$

Les deux calculs de disparité sur les marchés du film en salles dans les six pays européens étudiés montrent les faits suivants :

- la disparité paraît nettement plus importante en France que dans les autres pays, et ce au niveau de l'offre, de la distribution et de la demande. La hiérarchie entre les autres pays est plus floue. Au niveau de l'offre, ce sont les grands pays (Espagne et Royaume-Uni) qui portent le plus de disparité, mais le constat est moins évident en ce qui concerne la demande ;

- une tendance à la hausse de la disparité entre 1998 et 2004 se dessine sur tous les marchés lorsque l'on tient compte de l'indicateur de disparité par films (et non par catégories). Il montre une disparité de l'offre accrue entre 1998 et 2001 puis entre 2001 et 2004, et ce dans pratiquement tous les pays (à l'exception du Danemark). De même, il souligne une hausse globale de la disparité consommée entre 1998 et 2004, se décomposant entre une première hausse jusqu'en 2001, suivie d'une baisse de moindre envergure jusqu'en 2004.

\section{Un indicateur synthétique de diversité du marché du film en salles}

Il serait tentant de construire un indicateur ad hoc, à partir des indicateurs de variété, d'équilibre et de distance élaborés lors des sections précédentes. Mais Stirling a montré combien les indices de diversité existants peinaient à satisfaire aux exigences d'un bon indicateur. Dès lors, son indicateur synthétique de mesure de la diversité du marché a été repris. Il caractérise la diversité par la formule suivante (un résultat plus élevé est signe d'une plus grande diversité) :

$$
\mathrm{M}=\Sigma_{\mathrm{ij}} \mathrm{d}_{\mathrm{ij}} \cdot \mathrm{p}_{\mathrm{i}} \cdot \mathrm{p}_{\mathrm{j}}
$$

À partir des tableaux de fréquence et de la matrice de dissimilarité, et à l'aide de la formule de Stirling, les calculs de diversité pour chacun des six marchés des trois années peuvent être menés respectivement pour l'offre, la distribution et la demande.

Les diversités obtenues (tableau 2, graphiques 2, 3 et 4) semblent cohérentes par rapport aux différentes conclusions tirées de l'analyse des trois dimensions de la diversité : qu'il s'agisse de l'écart entre la diversité de l'offre et celle de la demande, du niveau de la diversité de la distribution, de l'évolution temporelle ou des variations géographiques, l'indice synthétique ne contredit pas l'analyse détaillée, une conclusion rassurante quant à la fiabilité de l'outil.

\section{Diversité de l'offre (films)}

La diversité de l'offre est supérieure en France, suivie du Royaume-Uni, du Danemark, de l'Espagne, de la Suède et enfin de la Pologne. Il est logique de retrouver un niveau supérieur de diversité en France lorsque l'on considère que pratiquement tous les indicateurs de variété, d'équilibre et de disparité ont montré que la France était le marché le plus varié, le plus équilibré ou encore le plus disparate.

L'outil ne place pas tous les grands marchés en tête, alors que plusieurs indicateurs, ceux exprimés en valeur absolue, les montraient plus riches, équilibrés ou disparates que les petits pays. Le Danemark, qui présentait en effet une offre très variée sur écrans et disparate lorsqu' on ramenait la distance au nombre de billets vendus vient, dans le classement final de diversité, s'intercaler entre le RoyaumeUni et l'Espagne.

Une tendance positive se dégage de l'évolution de la diversité de l'offre de films dans le temps. Cinq des six mar-

23. Voir à ce propos par exemple André LEMELIN, Méthodes quantitatives des sciences sociales appliquées aux études urbaines et régionales, Montréal, INRS, 2004.

24. Les catégories sont construites sur la base de quatre attributs. L'évaluation de la dissimilarité est établie entre chaque couple de catégories : un poids de 0 est attribué pour chaque attribut partagé par les deux catégories ; un poids de 1 est attribué pour chaque attribut non partagé par les deux catégories et un poids de 0,5 est attribué lorsque l'on ne peut comparer un attribut entre deux catégories (ainsi la structure de production n'est relevée que pour quatre des dix-neuf catégories).

25. Andrew STIRLING, "On the Economics and Analysis of Diversity", sPRU Electronic Working Paper no 28, 1998 (http://www.sussex.ac.uk/spru/research /sewps [consulté le 13 septembre 2011]). 


\begin{tabular}{|c|c|c|c|c|}
\hline & Diversité de l'offre & Diversité de la distribution $^{*}$ & \multicolumn{2}{|c|}{ Diversité de la demande } \\
\hline Tous films (tous pays et toutes années) & 1,102 & 0,907 & \multicolumn{2}{|l|}{0,751} \\
\hline 1998 (tous pays) & 1,067 & n.a. & \multicolumn{2}{|l|}{0,627} \\
\hline 2001 (tous pays) & 1,112 & 0,906 & \multicolumn{2}{|l|}{0,837} \\
\hline 2004 (tous pays) & 1,121 & 0,905 & \multicolumn{2}{|l|}{0,754} \\
\hline Danemark (toutes années) & 1,074 & n.a. & \multicolumn{2}{|l|}{0,82} \\
\hline DK 1998 & & 1,072 & n.a. & 0,67 \\
\hline DK 2001 & & 1,103 & n.a. & 0,874 \\
\hline DK 2004 & & 1,025 & n.a. & 0,835 \\
\hline Espagne (toutes années) & 1,042 & n.a. & \multicolumn{2}{|l|}{0,647} \\
\hline ES 1998 & & 1,021 & n.a. & 0,517 \\
\hline ES 2001 & & 1,053 & n.a. & 0,741 \\
\hline ES 2004 & & 1,059 & n.a. & 0,633 \\
\hline France (toutes années) & 1,168 & 0,918 & \multicolumn{2}{|l|}{0,879} \\
\hline FR 1998 & & 1,151 & n.a. & 0,70 \\
\hline FR 2001 & & 1,166 & 0,91 & 0,925 \\
\hline FR 2004 & & 1,178 & 0,914 & 0,909 \\
\hline Royaume-Uni (toutes années) & 1,108 & n.a. & \multicolumn{2}{|l|}{0,6} \\
\hline RU 1998 & & 1,061 & n.a. & 0,506 \\
\hline RU 2001 & & 1,092 & n.a. & 0,683 \\
\hline RU 2004 & & 1,128 & n.a. & 0,584 \\
\hline Pologne (toutes années) & 0,932 & 0,698 & \multicolumn{2}{|l|}{0,802} \\
\hline PL 1998 & & 0,854 & n.a. & 0,522 \\
\hline PL 2001 & & 0,995 & 0,75 & 0,979 \\
\hline PL 2004 & & 0,911 & 0,657 & 0,667 \\
\hline Suède (toutes années) & 1,008 & n.a. & \multicolumn{2}{|l|}{0,76} \\
\hline SE 1998 & & 0,933 & n.a. & 0,567 \\
\hline SE 2001 & & 0,99 & n.a. & 0,838 \\
\hline SE 2004 & & 1,04 & n.a. & 0,817 \\
\hline
\end{tabular}

Source : GEM-Sciences Po/DEPS, Ministère de la Culture et de la Communication, 2011.

Graphique 2 - Diversité de l'offre, de la distribution et de la demande en France en 1998, 2001 et 2004

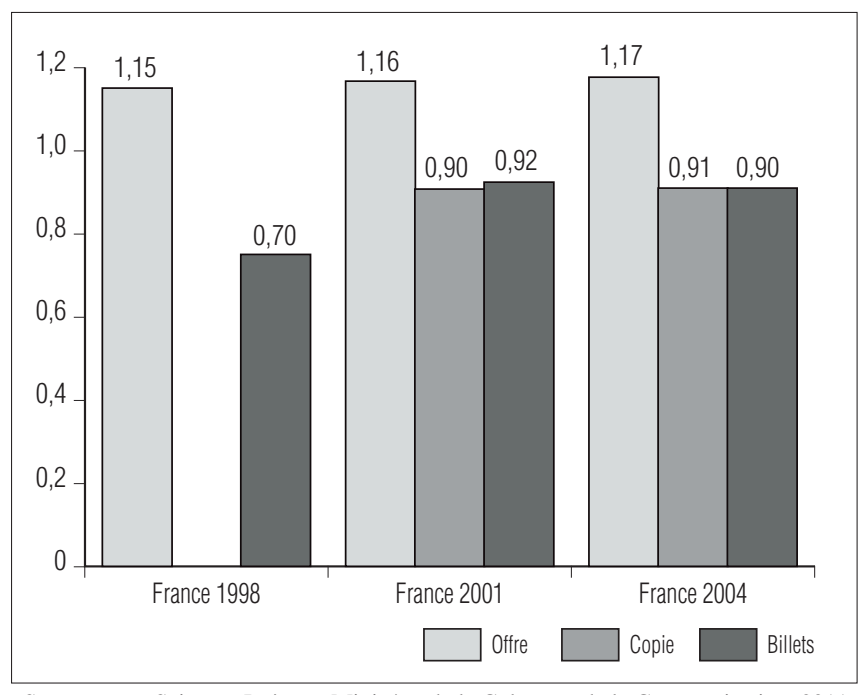

Source : GEM-Sciences Po/DEPS, Ministère de la Culture et de la Communication, 2011.

\section{Graphique 3 - Diversité de l'offre, de la distribution et de la demande en Pologne en 1998, 2001 et 2004}

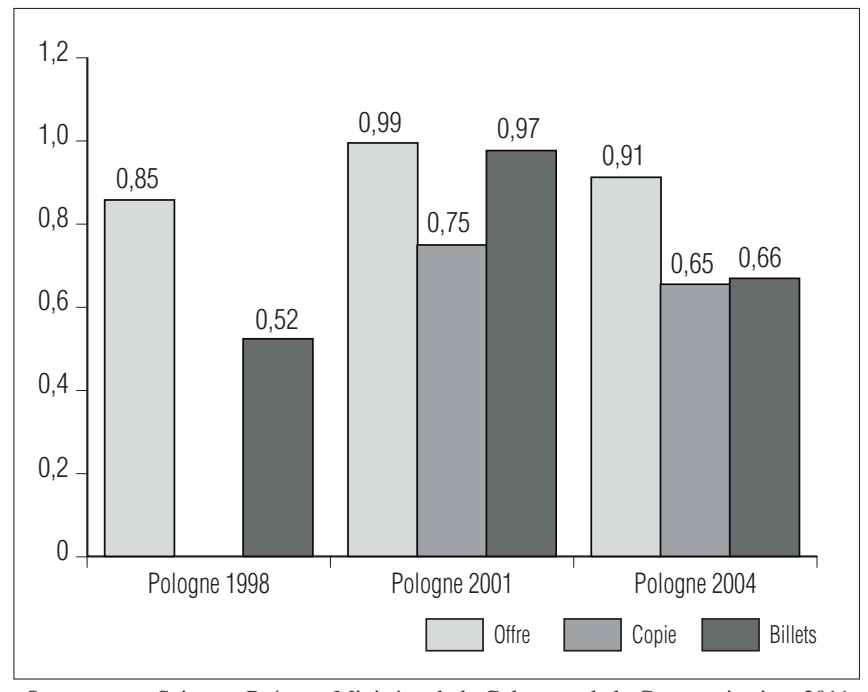




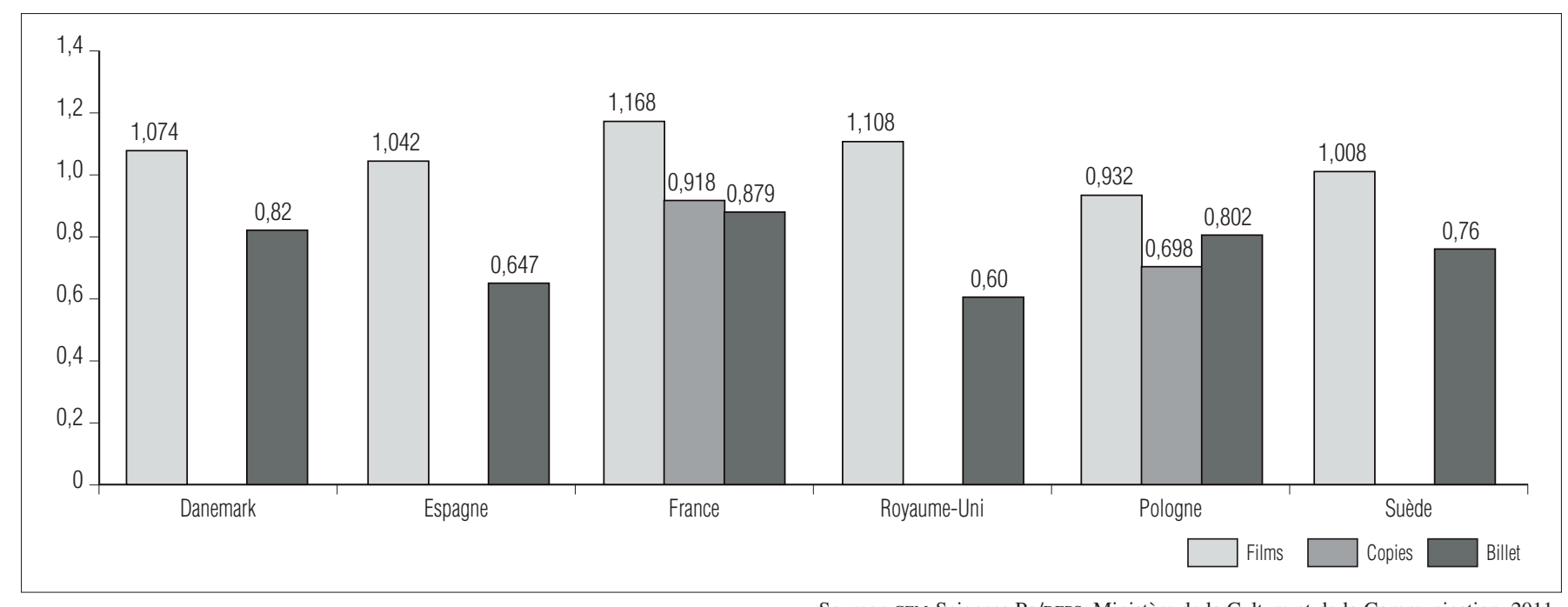

Source : GEM-Sciences Po/DEPS, Ministère de la Culture et de la Communication, 2011.

chés voient la diversité de leur offre progresser. Seule l'offre danoise paraît moins diverse en 2004 qu'en 2001 et même qu'en 1998, ce qui est cohérent par rapport aux résultats observés lors de l'analyse de la disparité des marchés.

\section{Diversité de la distribution (copies)}

La diversité de la distribution n'est calculée que pour deux marchés (France et Pologne) et deux années. Nettement inférieure à la diversité de l'offre pour les deux pays, la diversité des copies se révèle plus importante en France qu'en Pologne. La diversité des copies paraît stable en France entre 2001 et 2004 (de 0,91 à 0,914 ), mais diminue dans le même temps en Pologne (de 0,75 à 0,657).

\section{Diversité de la demande (billets vendus)}

C'est une nouvelle fois en France que la diversité demandée se révèle la plus importante $(0,879)$ suivie du Danemark, de la Pologne, de la Suède, de l'Espagne et enfin du Royaume-Uni. Si la diversité de l'offre est plutôt élevée dans les grands pays producteurs, cela ne garantit aucunement un niveau élevé de diversité demandée. Ainsi le cas du Royaume-Uni paraît-il exemplaire : second dans la hiérarchie de la diversité offerte $(1,11)$, il est dernier pour la diversité de sa demande ( 0,6 seulement).

La diversité de la demande progresse manifestement entre 1998 et 2004 et ce, grâce à une hausse spectaculaire (33\%) entre 1998 et 2001, qui n'est pas compensée par la baisse, moins marquée, enregistrée ensuite entre 2001 et $2004(0,754)$. Ce phénomène de très forte progression suivie d'une baisse jusqu'en 2004 est constaté sur les six marchés de l'échantillon. Bien sûr, il est difficile de conclure à une hausse structurelle de la diversité de la demande à partir de trois années seulement, puisque deux tendances s'opposent sur les deux périodes analysées, et que la demande paraît particulièrement volatile. On peut supposer que la hausse considérable de la diversité entre 1998 et 2001 fait suite au niveau exceptionnellement bas atteint par la diversité de la demande en $1998^{26}$.

\section{Synthèse des résultats}

Sur la base de nos résultats, la France est incontestablement le pays où le marché du film en salles connaît le plus haut degré de diversité. Ce constat s'applique tant à l'offre qu'à la distribution et à la demande.

La situation des deux autres grands marchés est moins prononcée puisqu'en Espagne comme au Royaume-Uni, on identifie une offre relativement diversifiée, mais une demande particulièrement concentrée.

Le rapprochement des niveaux de diversité des films, des copies et des billets sur les différents marchés montre, dans les six cas, un recul important des niveaux de diversité entre l'offre et la demande. L'écart est plus marqué entre la diversité offerte et celle distribuée qu'entre diversité distribuée et demandée, souvent très proches.

La diversité de l'offre progresse faiblement, mais régulièrement; la diversité de la distribution paraît plutôt stable ; enfin la diversité de la demande augmente fortement entre 1998 et 2001 puis régresse entre 2001 et 2004, avec une tendance globale toutefois positive mais peut-être due à une année 1998 «accidentelle » et exceptionnellement basse.

26. L'analyse des dimensions de la diversité a en effet montré combien les demandes sur les différents marchés ont été particulièrement concentrées et donc les marchés déséquilibrés en 1998, année du Titanic de James Cameron, qui a concentré $13 \%$ des entrées alors que les films les plus populaires de l'échantillon en 2001 et 2004 ne recueillent que 5 et 5,1\% des entrées (respectivement avec Harry Potter and the Philosopher's Stone et Schrek 2). Idéalement, il faudrait pouvoir étendre les calculs de diversité à un nombre plus important d'années pour pouvoir mettre en valeur cette hypothèse d'une année 1998 exceptionnellement basse. 


\section{LA DIVERSITÉ DU MARCHÉ DU FILM EN VIDÉOGRAMMES EN FRANCE}

La carrière d'un film ne se limite pas à son exposition sur les écrans de cinéma. Il est ensuite consommé à la maison selon la chronologie des médias : loué et vendu en vidéo, sous forme d'une vHs, d'un DVD, d'un Blu-ray, d'un fichier dématérialisé, ou encore diffusé à la télévision. Le marché des ventes de vidéogrammes, même s'il ne constitue que l'une des formes de consommation de films à la maison, présente plusieurs intérêts dans le cadre d'un travail sur la diversité des marchés cinématographiques.

\section{Disponibilié et collecte des données}

\section{La base de données GfK}

Le premier écueil à surmonter afin de cerner la diversité du marché de la vidéo est celui du manque de transparence de ce marché, les données de base, désagrégées, étant particulièrement problématiques à collecter.

Les instituts nationaux du film ne réalisent pas en Europe de collecte des données de vente au détail des vidéogrammes ${ }^{1}$. Face à l'évidente carence de données publiques s'affirme la nécessité de s'adresser à des fournisseurs privés de données. GfK décrypte de multiples marchés, parmi lesquels celui des biens culturels (livre, musique, vidéogrammes, loisirs interactifs, etc.) et s'affirme comme la seule organisation collectant des données de base sur le marché de la vidéo dans les différents pays européens. Son panel couvre plusieurs dizaines de milliers de références, si bien que les données GfK apparaissent, en France par exemple, comme les plus complètes ${ }^{2}$.

\section{Cadre géographique, temporel et économique}

L'absence de données publiques européennes, la nature des données de base fournies par GfK et les difficultés de traitement de ces données conduisent à limiter l'analyse du marché du film en vidéo au marché français et à deux années seulement : 2003 et 2005.

Enfin, deux mesures de diversité peuvent être établies : la diversité de l'offre, c'est-à-dire celle des vidéogrammes proposés à la vente, et la diversité de la demande, c'est-à-dire celle des vidéogrammes achetés par les consommateurs.

Une liste de 6528 films est établie qui représente l'intégralité des films offerts à la vente en vidéo en France en 2003 et 2005, et vendus à au moins 1000 unités $^{3}$. Pour chacun de ces films, une quinzaine d'attributs est collectée: nom du réalisateur, année de production, nationalité, langue, rang, budget de production, qualité, suite, présence de stars, producteur, distributeur, éditeur, nombre de copies, nombre de références, etc.

1. Si, en France, le CNC s'est doté d'un outil d'appréciation du marché des vidéogrammes, il fonctionne sur un mode déclaratif et ne couvre pas l'intégralité du marché.

2 . GfK recueille des données de ventes en sortie de caisse dans environ 3500 points de vente (en 2010) et extrapole ces données pour obtenir des données nationales.

3. L'application d'un tel seuil est naturellement dommageable à l'exhaustivité de l'échantillon, mais s'avère nécessaire, non seulement pour limiter le temps de collecte et de traitement des informations, mais aussi afin d'obtenir une liste de films la moins biaisée possible.
D'abord la diversité du marché du film en vidéo est moins souvent étudiée que celle du film en salles. Des données statistiques sont recueillies par exemple par le CNC, l'Observatoire européen de l'audiovisuel, Screen Digest et l'International Video Federation (IVF). Au-delà de ces données brutes et des descriptions traditionnelles du fonctionnement du marché, peu d'articles se sont réellement penchés sur la diversité du marché du film en vidéo (composition ou encore équilibre de l'offre ou des ventes, etc.). Ainsi la plupart des articles analysant les facteurs de succès des films, et donc la concentration de la demande de films sur quelques produits stars, se consacrent au succès en salles mais n'envisagent pas l'exploitation. L'analyse du phénomène dit de «longue traîne » a toutefois permis d'attirer davantage l'attention sur la disparité du marché de la vidéo.

Pourtant le marché de la vidéo représente un poids considérable au sein de la carrière des films et de l'économie du cinéma. Si les ventes de vidéogrammes équivalent à $55 \%$ des recettes en salles en France en 2000, cette part monte jusqu'à plus de $70 \%$ en 2008 (chiffres $\mathrm{CNC}$ ). $\mathrm{Au}$ niveau européen, le constat est similaire : en 2008 toujours, le chiffre d'affaire du marché de la vidéo au Royaume-Uni ou en Allemagne dépasse même celui de l'exploitation en salles (chiffres Observatoire européen de l'audiovisuel).

Enfin, le marché de la vidéo revêt un intérêt particulier à l'aune des débats relatifs à la numérisation et à la dématérialisation des œuvres. L'économie traditionnelle de la vidéo est triplement concernée. D'abord, parce que les DVD, vHS ou Blu-ray s'achètent de plus en plus sur des plates-formes de distribution en ligne. Ensuite, parce que les films peuvent désormais se louer ou s'acheter non plus sous la forme d'un produit physique (DVD, VHS, etc.) mais grâce à un simple téléchargement (ou par streaming) de fichier électronique (vidéo à la demande). Enfin, parce que les fichiers s'échangent de plus en plus facilement, circulent d'un utilisateur à l'autre, en partie illégalement.

\section{Variété, équilibre et disparité du marché du fillm en vidéo}

\section{Variété du marché du film en vidéogrammes}

La variété, ou richesse, peut représenter l'étendue des choix proposés sur le marché (variété de l'offre) ou l'étendue des achats des consommateurs (variété de la demande). Deux indicateurs de variété utilisés sur le marché du film en salles sont repris ${ }^{27}$ :

- le nombre d'espèces présentes dans la collection ;

- le nombre de produits présents dans la collection.

Les deux indicateurs mènent à un double constat :

- la variété (de l'offre et de la demande) progresse entre 2003 et 2005. C'est ainsi que le nombre de vidéogram-

27. Les deux indicateurs de variété fondés sur des nombres de produits pondérés, utilisés lors de l'analyse de la diversité du marché du film en salles, ne sont pas réutilisés dans le cadre de l'analyse de la variété du marché du vidéogramme. La pondération par le nombre d'écrans utilisée sur le marché du film en salles n'est évidemment pas pertinente dans le cadre de l'analyse du marché de la vidéo et la pondération par la population ne présente aucun intérêt en l'absence de comparaisons internationales. 


\section{Réutilisation de la nomenclature de films}

Les données ainsi rassemblées doivent permettre d'élaborer une mesure de la diversité des marchés du film en vidéo. À cette fin, la méthodologie testée sur le marché du film en salles est reprise: les films sont répartis dans une nomenclature, faisant écho à la démarche biologique de classification des espèces. Cette nomenclature sert de support aux analyses des trois dimensions de la diversité, envisagées successivement, puis de manière synthétique.

La nomenclature de films est identique à celle de l'analyse de la diversité du marché du film en salles (voir supra). La distinction entre les catégories de films se fonde donc toujours sur quatre critères : l'ancrage culturel du film (nationalité et langue parlée), la structure de production (majors ou producteurs indépendants pour les films américains), l'exigence artistique/la qualité et enfin l'âge du film. Ce dernier critère pose toutefois problème.

Le poids des films de patrimoine dans l'échantillon de 6528 films sur le marché des vidéogrammes s'avère considérable. C'est ainsi que les films de plus de trois ans (définis comme films de patrimoine) représentent près de $75 \%$ de l'échantillon. Contrairement au marché du film en salles, le marché du film en vidéogrammes n'est pas essentiellement organisé autour de la nouveauté. Le marché du film en vidéo n'est pas un marché de stock et les films ne disparaissent pas des étals des magasins après quelques semaines comme ils ne sont plus à l'affiche des salles de cinéma. Regrouper tous les films de patrimoine dans une catégorie unique revient à biaiser les calculs: l'équilibre entre les catégories sera alors sous-évalué (car une catégorie va regrouper les trois quarts des films) et la disparité sera elle surévaluée (puisque ces films très nombreux seront considérés comme très éloignés des autres). La seule variante par rapport à la méthode utilisée sur le marché du film en salles consiste dans la suppression du critère d'âge des films et de la catégorie dédiée aux films de patrimoine ${ }^{1}$. Le critère d'âge du film n'est plus pris en compte dans l'analyse, mais cela ne signifie pas que les films de patrimoine soient exclus de l'analyse. Celle-ci porte toujours sur les 6528 films de l'échantillon, mais tous les films de patrimoine sont requalifiés, basculant dans l'une des dix-huit autres catégories selon leur origine, leur structure de production et leur qualité.

Le recueil de données et l'élaboration des tableaux d'effectifs et de fréquences permettent la mise en place des indicateurs de variété, d'équilibre, de disparité, et enfin de diversité, sur ce marché méconnu qu'est le film en vidéogrammes. Leur analyse doit permettre de déterminer, notamment, si la diversité du marché du film en vidéogrammes est comparable avec celle du film en salles et si elle varie selon les plates-formes de distribution: la diversité des œuvres achetées sur l'internet, par exemple, estelle supérieure à celle des œuvres achetées en magasins traditionnels?

1. Notons que cette catégorie (appelée " 9 ») ne se trouve pas pour autant complètement vide car celle-ci regroupait les films de patrimoine et les films muets. Ces derniers demeurent donc dans la catégorie « 9 ». mes différents offerts sur le marché passe de 2650 films en 2003 à 3880 en $2005^{28}$ et que le volume des ventes augmente de 43 à 54 millions entre 2003 et 2005 en France ;

- l'offre apparaît plus variée en vidéo qu'en salles, mais c'est en salles que la demande est la plus variée. Les deux indicateurs montrent la plus grande variété de l'offre sur le marché du film en vidéogrammes. Alors qu'en France, en 2001 ou en 2004, seules 17 catégories sont représentées, 19 sont présentes sur le marché du film en vidéo en 2003 et en 2005. Surtout, un peu plus de 500 films sont projetés par an dans les salles françaises, quand plus de $3000^{29}$ sont disponibles sur le marché de la vidéo. En revanche, le constat est inverse pour la demande. Ainsi, le nombre de films vus dans les salles en une année dépasse nettement le nombre de films achetés en vidéo: entre 154 et 177 millions de billets vendus contre 43 et 54 millions de vidéogrammes vendus.

\section{Équilibre du marché du film en vidéogrammes}

L'équilibre du marché concerne l'importance respective des différentes catégories de films dans l'offre et la demande de vidéogrammes, c'est-à-dire la plus ou moins égale répartition des films entre les catégories.

La série de cinq indicateurs d'équilibre utilisés pour le marché du film en salles est reprise :

- deux indices de dominance (indice de Berger-Parker et coefficient de concentration);

- deux mesures de l'équilibre seul (indices de McIntosh et d'Herfindahl-Hirschman);

- un indice dual, tenant compte à la fois de l'équilibre et de la variété (indice dit de diversité de McIntosh).

Les différents calculs permettent de tirer les conclusions suivantes :

- l'offre de vidéogrammes s'avère mieux équilibrée que la demande, et ce quel que soit l'indicateur retenu. Les deux indices de dominance montrent que la ou les catégories dominantes rassemblent une part moindre de l'offre par rapport à la demande. Par exemple, les trois catégories dominantes ${ }^{30}$ réunissent $57 \%$ des films offerts en vidéo, mais $69 \%$ des ventes. L'indicateur d'Herfindahl-Hirschman indique aussi un meilleur équilibre de l'offre que de la demande: 1499 sur la moyenne des années pour l'offre et 2193 pour la demande. On constate donc que l'offre se situe en deçà de la barre symbolique des 2000 , souvent interprétée comme un seuil de concentration sur un marché, tandis que la demande se situe au-delà ;

28. La hausse de la variété offerte semble logique puisque le marché du film en vidéogrammes fonctionne sur un mode cumulatif (plus de films en vidéogrammes sont disponibles en 2005 qu'en 2003). C'est un marché de stock, contrairement au marché du film en salles, marché de la nouveauté où la variété est naturellement limitée par le nombre d'écrans et de semaines dans l'année. Les films en vidéogrammes restent quant à eux disponibles dans les bacs des magasins de vente au détail pendant des mois, voire des années, et ce d'autant plus facilement lorsque les magasins se dématérialisent à travers le commerce en ligne.

29. Ce chiffre ne concerne que les films vendus à plus de 1000 exemplaires, qui représentent néanmoins $96 \%$ des ventes sur le marché du vidéogramme.

30. Les trois catégories qui sont les mieux représentées dans l'offre de films en vidéogrammes sont aussi celles qui dominent la demande sur ce marché. Il s'agit des catégories « $100 »($ Catwoman), «110» (Psycho beach party) et « $30 »$ (Vidocq), c'est-à-dire trois catégories de films à faibles prétentions artistiques. On note donc un écart par rapport au marché du film en salles : les chiffres du marché français montraient en effet que les catégories dominantes dans l'offre étaient les « $100 »$ (Catwoman), « $30 »$ (Vidocq) et « 9 » (Les 400 coups), et dans la demande les catégories « $100 »($ Catwoman), «30» (L’Anglaise et le duc) et «31» (Vidocq). Les films domestiques de qualité sont donc relativement plus importants sur le marché du film en salles par rapport à celui de la vidéo. 
- l'équilibre progresse entre 2003 et 2005 . Les cinq indicateurs montrent un meilleur équilibre, tant de l'offre que de la demande, en 2005 par rapport à 2003 ;

- le marché du film en vidéo paraît moins équilibré que celui du film en salles du point de vue de l'offre, mais pas de la demande. Le constat est intéressant car le marché du film en vidéogrammes est souvent présenté comme moins porteur de diversité, plus uniforme et moins original que le marché du film en salles. En particulier, la demande de films en vidéogrammes est souvent décrite comme particulièrement concentrée, se portant encore plus massivement que celle du film en salles vers quelques titres, souvent peu originaux et de médiocre facture. Si les indicateurs d'équilibre ne nous disent encore rien à propos de la disparité sur le marché du film en vidéo, ils pointent en revanche une demande finalement moins concentrée que sur le marché du film en salles.

\section{Disparité du marché du film en vidéogrammes}

Les films des différentes catégories sont-ils plus ou moins similaires les uns aux autres ? La distance entre les films, notion complexe et peu aisée à quantifier, est ici envisagée comme degré de dissimilarité entre les catégories de films. Sur cette base, la demi-matrice de dissimilarité ${ }^{31}$, élément central permettant les calculs de disparité décrite lors de l'étude du marché du film en salles, est reprise.

Sur le fondement des dissimilarités entre les catégories de films, deux indicateurs de disparité sont testés :

- la distance totale entre les catégories présentes dans l'échantillon ;

- la distance totale entre les films présents dans l'échantillon.

Le second indicateur est articulé autour des produits et non plus uniquement des catégories. Il reflète par conséquent plus que la seule disparité sur le marché puisqu'il rend aussi compte de la variété (en nombre de produits).

Les calculs mènent à une série de constats sur le marché de la vidéo en France :

- la disparité de la demande n'apparaît pas comme inférieure à celle de l'offre. Selon le premier indicateur, la distance entre les films offerts et consommés ne varie pas ou presque, ce qui est dû au fait que le nombre de catégories représentées est le même pour l'offre et la demande. En revanche, selon le second indicateur (la distance totale entre les films), la distance consommée est nettement supérieure à la distance offerte. Ainsi, dans le cas de l'offre, la distance totale prend en compte la distance entre tous les films de la liste, tandis que dans le cas de la demande, il s'agit de la distance entre toutes les unités vendues. La variété (en produits) entre donc en jeu dans ce calcul, et favorise indéniablement une disparité élevée de la demande ;
- la disparité progresse entre 2003 et 2005, selon l'indicateur de distance entre produits. Mais l'augmentation de la distance totale, sensible aux variations de la variété offerte et demandée, est logique, puisque l'on a déjà noté une hausse sur cette période des variétés de l'offre et de la demande;

- la disparité s'avère plutôt supérieure à celle du marché du film en salles au regard de l'indicateur de distance entre les catégories. La distance totale entre les films désigne quant à elle l'offre du marché de la vidéo comme plus disparate que celle du marché du film en salles, mais aboutit à une conclusion inverse lorsqu'il s'agit de la demande. Mais, comme nous l'avons indiqué, le premier indicateur ne semble pas particulièrement pertinent dans le cadre de notre nomenclature limitée de film, et le second est trop sensible aux différences de variété entre les marchés.

\section{Un indicateur synthétique de diversité du marché du film en vidéogrammes}

La répartition des films en catégories et la matrice de dissimilarité entre ces catégories de films permettent d'appliquer la formule élaborée par Stirling, en d'autres termes de calculer un indice de diversité du marché, tenant compte à la fois de la variété, de l'équilibre et de la disparité sur le marché.

Peu transparent, le marché de la vidéo est finalement peu étudié, et la diversité de ce marché n'a jamais encore été envisagée de manière synthétique. Le calcul de diversité permet de dresser une série de constats.

\section{Tableau 3 - Indices de diversité de l'offre et de la demande de vidéogrammes}

\begin{tabular}{|lcc|}
\hline & Diversité de l'offre & Diversité de la demande \\
\hline Tous films & 0,93 & 0,79 \\
2003 & 0,91 & 0,75 \\
2005 & 0,94 & 0,83 \\
\hline
\end{tabular}

Source : GEM-Sciences Po/DEPS, Ministère de la Culture et de la Communication, 2011

\section{Une diversité de l'offre supérieure à celle de la demande}

Conformément aux résultats des indicateurs de variété, d'équilibre et d'une partie des indicateurs de distance, l'offre apparaît finalement sensiblement plus diverse que la demande. L'indicateur passe ainsi de 0,93 pour l'offre à 0,79 seulement pour la demande.

\section{Plus de diversité en 2005 qu'en 2003}

Le niveau de diversité obtenu est supérieur en 2005 par rapport à 2003, ce qui est cohérent avec les analyses des

31. Étant donné la priorité accordée à la comparaison entre les différents marchés, la matrice n'a pas dû être modifiée et les distances entre les catégories ont pu être maintenues à l'identique par rapport à l'analyse du marché du film en salles. 


\section{Graphique 5 - Indices de diversité de l'offre et de la demande de films en salles et en vidéogrammes}

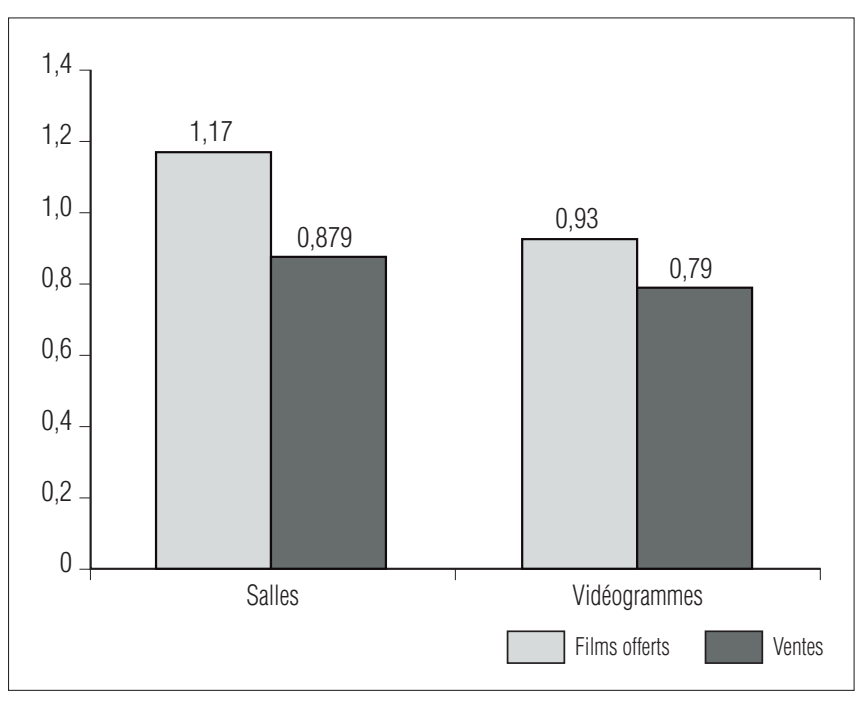

Source : GEM-Sciences Po/DEPS, Ministère de la Culture et de la Communication, 2011.

différentes dimensions de la diversité développées dans les sections précédentes. Celles-ci établissaient en effet à la fois une variété accrue ${ }^{32}$, un meilleur équilibre et plus de disparité en 2005 par rapport à 2003, pour l'offre comme pour la demande. C'est donc logiquement que l'offre passe d'un niveau de diversité de 0,91 à 0,94 et que la demande progresse de 0,74 à 0,83 .

On note ici que la diversité de la demande progresse dans des proportions plus importantes que la diversité de l'offre $(12,2 \%$ contre $3,3 \%)$. Bien entendu, cela ne permet pas de tirer des conclusions en termes d'évolution dans le temps du marché du film en vidéogrammes.

\section{Films en salles et films en vidéogrammes}

Un premier constat réside dans le fait que les deux marchés connaissent des niveaux de diversité relativement proches, et donc remarquablement comparables. Il faut rappeler cependant que la possibilité de comparer entre les niveaux de diversité des deux marchés (film en salles et film en vidéogrammes) doit être nuancée, les deux calculs ne s'appuyant pas sur des méthodologies absolument harmonisées : en particulier, le traitement des films de patrimoine n'est pas similaire sur les deux marchés, puisqu'ils forment une catégorie spécifique pour l'analyse du marché du film en salles, mais pas pour celui du film en vidéogrammes.

Malgré cette réserve, que nous enseigne le rapprochement des niveaux de diversités mesurés en France sur les marchés du film en salles et du film en vidéogrammes ?

La diversité apparaît supérieure sur le marché du film en salles, tant pour l'offre que pour la demande. Mais l'écart entre les deux marchés est surtout marqué au niveau de l'offre $(1,17$ contre 0,93$)$. En effet, les niveaux respectifs de diversité des demandes s'avèrent proches : 0,88 pour le film en salles et 0,79 pour le film en vidéogrammes (graphique 5). Ce constat implique bien sûr que l'écart entre l'offre et la demande est supérieur sur le marché du film en salles par rapport à celui qui caractérise le marché du film en vidéo. Cet écart moindre entre la diversité de l'offre et de la demande est vraisemblablement lié aux caractéristiques structurelles du marché de la vidéo par rapport à celui du film en salles : l'intermédiation est sensiblement plus fluide sur le marché des vidéogrammes que sur celui du film en salles, ce qui facilite d'autant l'appariement entre l'offre et la demande finale.

L'absence de données relatives à la distribution sur le marché du film en vidéogramme ne permet pas de comparer intégralement les calculs de diversité menés sur le marché du film en salles.

Le marché du film en salles et celui du film en vidéogrammes sont structurés différemment, la distribution des copies dans les salles de cinéma n'ayant rien à voir avec celle des vidéogrammes dans les différents points de vente. À défaut de données sur la distribution de vidéogrammes (les tirages des films), pour ne pas délaisser l'analyse du maillon de la distribution, et sur le fondement de la base GfK qui livre un ensemble de données sur les offres et les ventes en fonction des différents points de vente des vidéogrammes, il est intéressant de mener une nouvelle vague de calculs de la diversité du marché du film en vidéogrammes selon le type de points de vente (ou type de circuit de distribution).

\section{Diversité du marché des films en vidéogrammes selon le circuit de distribution}

La base de données GfK permet de construire une seconde série d'extractions de films en vidéogrammes, qui autorise des comparaisons de la diversité (de l'offre et de la demande) entre les différents circuits de distribution pour l'année $2005^{33}$. Les données par circuit de distribution sont structurées autour de quatre circuits :

- les grandes surfaces spécialisées (GSS), qui regroupent les enseignes telles que la Fnac, Virgin, etc.;

- les grandes surfaces alimentaires (GSA), comme par exemple les espaces culturels Leclerc, Auchan, etc.;

- l'internet;

- les modes résiduels de distribution (autres), définis comme les maisons de la presse, la vente par correspondance ou encore les soldeurs.

La nomenclature des films décrite dans la section précédente est réutilisée. La répartition des films dans les différentes catégories permet de construire de nouveaux tableaux d'effectifs et de fréquences, par circuit de distribution, qui vont constituer le fondement d'une nouvelle

32. En particulier, la variété des produits offerts progresse sensiblement entre 2003 et 2005, ce qui pourrait s'expliquer par le fonctionnement cumulatif du marché de la vidéo et par l'essor du commerce par l'internet.

33. Le fait que les ventes par l'internet ne soient pas représentatives dans la base GfK en 2003 pousse à ne retenir que l'année 2005 pour l'analyse de la diversité selon le circuit de distribution. 
série de calculs de variété, équilibre, distance et finalement diversité.

La combinaison des trois dimensions de la diversité par circuit de distribution est opérée à travers la formule pré-

\section{Tableau 4 - Diversités de l'offre et de la demande selon le circuit de distribution, année 2005}

\begin{tabular}{|lcc|}
\hline & Diversité de l'offre & Diversité de la demande \\
GSS & 0,950 & 0,92 \\
GSA & 0,87 & 0,75 \\
Internet & 0,949 & 0,90 \\
Autres & 0,84 & 0,80 \\
\hline
\end{tabular}

sentée par Stirling et déjà utilisée dans la section précédente (tableau 4).

Une série de conclusions peut être tirée des calculs de diversité par circuit de distribution.

\section{Des demandes moins diversifiées que les offres, en particulier en GSA}

D'abord, il émerge que les diversités des demandes sont inférieures aux diversités des offres sur les quatre réseaux de distribution. Mais l'écart entre l'offre et la demande est le plus sensible dans les GSA, puisque le niveau de diversité se situe à 0,87 pour l'offre, mais seulement 0,75 pour la demande.

\section{GSS et l'internet versus GSA et autres circuits de distribution}

Surtout, la diversité des films offerts et demandés est la plus importante en GSS, juste devant l'internet. Les deux circuits de distribution présentent des niveaux de diversité proches. Derrière ces deux circuits se trouvent les GSA puis les autres points de vente. Un fait essentiel s'impose donc : la nécessité de distinguer entre d'un côté les GSS et l'internet et de l'autre les GSA et les autres circuits de distribution.

La diversité est supérieure sur les deux premiers circuits, par rapport aux deux derniers : en GSS et sur l'internet, les films offerts sont plus nombreux, l'offre plus équilibrée et les films présentés plus différents les uns des autres que sur les deux dernières plates-formes, où l'offre est à la fois moins riche, moins équilibrée et moins disparate. L'écart de diversité entre les deux types de circuits de distribution est encore plus net au niveau de la demande.

\section{La diversité des vidéos en GSS et sur l'internet}

Les GSS et l'internet présentent donc des offres et des demandes de films aux caractéristiques finalement très proches : films aux origines plus variées, en particulier films de pays tiers, tournés dans des langues autres que l'anglais ou le français, films de meilleure qualité, plus souvent indépendants ou encore plus souvent de patrimoine.
La principale différence entre les deux circuits est identifiée au niveau de la variété de la demande, plus élevée en GSS que sur l'internet, ce qui traduit le niveau encore faible des ventes sur l'internet. Hormis ce point, les offres de films en GSS et sur l'internet se révèlent largement comparables du point de vue de la variété, de l'équilibre et de la disparité, et les demandes apparaissent similaires au regard de l'équilibre et de la disparité.

\section{Le marché des vidéogrammes sur l'internet}

Quelques constats peuvent être formulés à propos du marché de la vidéo sur l'internet : d'abord, l'internet présente un catalogue de films relativement étendu alors que sa demande paraît encore relativement faible en 2005 (le catalogue offert est comparable à celui des GSS pour des ventes près de trois fois inférieures). Ensuite, les résultats des calculs de diversité confirment l'hypothèse d'un réseau de distribution plutôt divers, mais sans toutefois prouver qu'il propose une diversité supérieure à celle que l'on trouve dans les circuits traditionnels, à tout le moins en 2005. En somme, le développement du commerce de vidéogrammes par l'internet semble loin de présenter une menace pour la diversité du marché, bien au contraire. Cela dit, les données datent de 2005 et nécessiteraient d'être réactualisées. Ainsi, il est probable que la demande de films par l'internet reflète les caractéristiques sociologiques des publics fréquentant les sites de vente en ligne de vidéos (populations plus jeunes, diplômées ou technophiles par exemple) mais que ces caractéristiques évoluent avec le temps et sous l'effet de la démocratisation des usages de commerce en ligne notamment.

\section{La faible diversité des films offerts et surtout vendus en GSA}

Face aux GSS et à l'internet se trouvent enfin les GSA et les autres circuits de distribution, faiblement diversifiés. Ils se caractérisent par une forte présence des films américains, en langue anglaise, plutôt de mauvaise qualité, produits par des entreprises intégrées et/ou distribués dans les salles françaises par des filiales de studios américains ou enfin des suites.

Si l'offre des GSA est faiblement diversifiée, ce sont surtout les ventes en GSA qui se portent sur les films uniformes et peu divers. Le constat est d'autant plus inquiétant que ce réseau représente le circuit de distribution de loin le plus important en France, avec la moitié des ventes de vidéogrammes en France en 2005.

\section{Conclusion}

Les indicateurs synthétiques de diversité permettent de tirer des enseignements en termes géographiques, temporels, d'envisager plusieurs marchés, et d'appréhender les différents maillons à l'intérieur d'un même marché.

Pour autant, l'indicateur de diversité présenté ici l'est à titre exploratoire et demande naturellement à être amélioré : d'une part, parce que la méthode testée repose sur des partis pris subjectifs qui peuvent être discutés et conditionnent 
directement les résultats présentés ; d'autre part, parce que l'indicateur présente les degrés de diversité des marchés cinématographiques, par pays et par année, mais ne prétend pas fournir les explications aux divergences constatées. La mesure de la diversité vise en effet seulement à poser des diagnostics, ce qui constitue une première étape avant l'analyse de l'origine des écarts, qui représente une vague de recherches complémentaires.

La démarche s'inscrit dans un contexte de montée des préoccupations et des besoins internationaux en termes de statistiques culturelles ${ }^{34}$ et souligne, au-delà du degré de diversité des marchés du cinéma en Europe, la diversité des méthodologies de mesure de cette diversité.

Articulé autour d'une nomenclature subjective de films multidimensionnelle, d'une conception de la distance entre les films en termes de dissimilarité et de la combinaison entre les trois dimensions de la diversité (variété, équilibre et disparité) par la formule de Stirling, l'indicateur synthétique de diversité permet une lecture immédiate et aisée des résultats en termes de diversité des marchés. Il favorise la prise de conscience, facilite l'arbitrage et la prise de décision et autorise une évaluation des politiques mises en œuvre. De fait, un outil de mesure de la diversité s'avère

\begin{abstract}
Quatre principales recherches ont été menées jusqu'à présent visant à mesurer la diversité de marchés culturels : la première porte sur trois produits (disque, film et livre) dans huit espaces géographiques en Europe, Asie, Afrique, Amérique du Nord et du Sud (Stéphanie Peltier, François Moreau, Propositions exploratoires de mesures quantitatives de la diversité culturelle, document de travail pour la Direction générale de la coopération internationale et du développement, Paris, ministère des Affaires étrangères, 2002), la deuxième sur le film dans six pays (id., "Cultural Diversity in the Movie Industry: A Cross-National Study", Journal of Media Economics, 2004, vol. 17, n² 2, p. 123143), la troisième sur le marché du livre en France (Françoise Benhamou, Stéphanie Peltier, «Une méthode multicritères d'évaluation de la diversité culturelle : application à l'édition de livres en France ", dans GrefFe Xavier (sous la dir. de), Création et diversité au miroir des industries culturelles, Paris, ministère de la Culture et de la Communication/DEPS, coll. «Questions de culture », 2006, p. 313-344) - voir aussi, des mêmes auteurs, "How Should Cultural Diversity Be Measured? An Application Using from Publishing Industry", Journal of Cultural Economics, juin 2007 - et la dernière sur l'édition de livres dans 20 pays de l'OCDE entre 1979 et 2005 (Françoise BenHAmou, Renato G. Flores, Stéphanie Peltier, Diversity Analysis in Cultural Economics: Theoretical and Empirical Considerations, document de travail du GRANEM, $\mathrm{n}^{\circ}$ 2009-05-17, université d'Angers, septembre 2009, sur : http://ead.univ-angers.fr/ grane m08/spip.php?rubrique36 [consulté le 1er juillet 2010]). De plus, un groupe d'experts sur la mesure statistique de la diversité des expressions culturelles s'est réuni à deux reprises, dans le cadre de l'Unesco, en particulier à Barcelone en décembre 2008 où il a débattu de la mesure de la diversité des expressions culturelles.
\end{abstract}

indispensable à l'identification des menaces pesant sur la diversité d'un marché, à la détermination (ou à la justification) d'une politique économique ou culturelle adaptée.

Sans doute, la démarche de mesure de la diversité des marchés audiovisuels peut choquer par son aspect réducteur: la diversité culturelle est une riche idée, une notion pleine de sens, sans doute trop pleine de sens. Mais au final, ce sont bien les travaux de définitions, d'objectivation du concept et le travail de mesure de la diversité des marchés culturels qui représentent l'unique voie pour pérenniser et crédibiliser l'idée de diversité culturelle.

\section{ÉLÉMENTS DE BIBLIOGRAPHIE}

Anderson Chris, "The Long Tail", Wired Magazine, octobre 2004, $\mathrm{n}^{\circ} 12-10$, p. 170-177.

Anderson Chris, The Long Tail: Why the Future of Business is Selling Less of More, New York, Hyperion, 2006, 256 p.

BENHAMOU Françoise, «La diversité culturelle : regards d'une économiste sur un concept trop rassembleur pour être tout à fait honnête », Mouvements, janvier 2005, vol. 37, nº 1, p. 8-14.

Benhamou Françoise, Peltier Stéphanie, "How Should Cultural Diversity Be Measured? An Application Using the French Publishing Industry", Journal of Cultural Economics, juin 2007, vol. $31, \mathrm{n}^{\circ} 2$, p. 85-107.

BERNIER Ivan, ATKINSON Dave, «Mondialisation de l'économie et diversité culturelle: les arguments en faveur de la préservation de la diversité culturelle », Document de réflexion préparé pour la $2^{\mathrm{e}}$ concertation intergouvernementale de l'agence intergouvernemental de la francophonie, Paris, 2000. Disponible sur : http://www.agence.francophonie.org/diversiteculturelle/fichiers/a if_mondialisation_eco.pdf [consulté le $1^{\text {er }}$ juillet 2010].

CNC, Bilans annuels, Paris, CNC [1962-2010].

EIDE Asbjørn, "Cultural Rights as Individual Human Rights", dans EIDE et al. (sous la dir. de), Economic, Social and Cultural Rights, a Textbook, Leiden : Kluwer Law International, 2001, p. 9-28.

Elberse Anita, Oberholzer-Gee Felix, "Superstars and Underdogs: an Examination of the Long Tail Phenomenon in Video Sales", Working Paper $n^{\circ}$ 07-015, Harvard Business School, 2006. Disponible sur : http://www.people.hbs.edu/aelberse/papers/hbs_07-

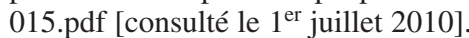

Ivf, European Video Market: The Industry Overview, Londres, Ivf [2003-2009].

LEMELIN André, Méthodes quantitatives des sciences sociales appliquées aux études urbaines et régionales, Montréal, INRS, 2004.

Nikoltchev Susanne, Éditorial, dans Nikoltchev Susanne (sous la dir. de), la Promotion de la diversité culturelle grâce aux nouvelles technologies des médias : introduction aux défis opérationnels, Strasbourg, Observatoire européen de l'audiovisuel, 2008, p. 1.

ObSERVATOIRE EUROPÉEn DE L'AUdiovisuel, Annuaire, Strasbourg, Observatoire européen de l'audiovisuel [1994-2010].

RAVID S. Abraham, "Information, Blockbusters, and Stars: a Study of the Film Industry", The Journal of Business, octobre 1999, vol. $72, \mathrm{n}^{\circ} 4$, p. $463-492$.

SCREEN Digest, European Video Yearbook, Londres, SCREEN Digest/Ivf [-2009].

Unesco, Convention sur la protection et la promotion de la diversité des expressions culturelles, CLT-2005/CONVENTION DiversiteCult Rev, Paris, 2005. Disponible sur : http://unesco.org/images/ 0014/001429/142919f.pdf [consulté le 1 er juillet 2010].

34. Plusieurs dispositions de la Convention sur la promotion et la protection de la diversité des expressions culturelles (Unesco, 2005) soulignent le besoin d'indicateurs statistiques culturels au niveau international. Si l'hypothèse de la création d'un observatoire de la diversité des expressions culturelles n'a pas été retenue par la version finale de la Convention, l'article 19 insiste toutefois sur l' 'échange, l'analyse et la diffusion de l'information ». Par ailleurs, des réunions d'experts sur la mesure statistique de la diversité des expressions culturelles sont régulièrement organisées sous l'égide de l'Unesco. Enfin, plusieurs autres articles de la Convention posent la question de la statistique culturelle, comme par exemple l'article 9 «Partage de l'information et transparence » qui définit les rapports que les Parties doivent adresser tous les quatre ans à l'Unesco : ceux-ci devront-ils et pourront-ils inclure des statistiques sur la diversité des marchés et sur les menaces pesant sur des expressions culturelles? 


\begin{tabular}{|c|c|c|c|}
\hline $\begin{array}{l}\text { études } \\
\text { quantitatif s'accompagne o } \\
\text { de la consommation? Fon } \\
\text { nelle d'Andrew Stirling, la } \\
\text { est analysée sur trois segm } \\
\text { dessinées, littérature) selon } \\
\text { duite et consommée, l'équil } \\
\text { titres et la disparité des ou } \\
\text { apporte des éléments de rél } \\
\text { de la loi sur le prix unique } \\
\text { et sur l'effet de levier des } \\
\text { longue traîne). }\end{array}$ & $\begin{array}{l}\text { CE-2011-4 } \\
\text { La diversité } \\
\text { culturelle } \\
\text { dans l'industrie } \\
\text { du livre en France } \\
\text { (2003-2007) } \\
\text { Octobre 2011, 16 p. } \\
\text { Si la production éditoriale } \\
\text { française a beaucoup progressé } \\
\text { au cours des années } 2000 \text { en } \\
\text { termes de titres et d'exem- } \\
\text { plaires produits annuellement, } \\
\text { comment apprécier si ce bond } \\
\text { non d'une plus grande diversité } \\
\text { ée sur l'approche tridimension- } \\
\text { mesure de la diversité culturelle } \\
\text { nts éditoriaux (jeunesse, bandes } \\
\text { rois dimensions : la variété pro- } \\
\text { ore des ventes entre les différents } \\
\text { res et des auteurs lus. L'analyse } \\
\text { onse à la question de l'efficacité } \\
\text { en termes de diversité culturelle } \\
\text { entes en ligne (hypothèse de la }\end{array}$ & 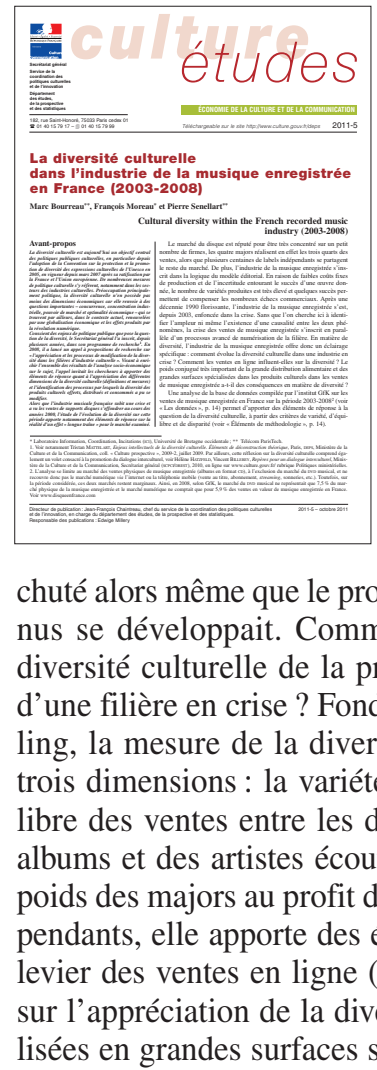 & $\begin{array}{l}\text { CE-2011-5 } \\
\text { La diversité } \\
\text { culturelle } \\
\text { dans I'industrie } \\
\text { de la musique } \\
\text { enregistrée } \\
\text { en France } \\
\mathbf{( 2 0 0 3 - 2 0 0 8 )} \\
\text { Octobre 2011, 16 p. } \\
\text { L'industrie musicale française } \\
\text { est en crise depuis le milieu des } \\
\text { années 2000. Les ventes ont } \\
\text { cessus de numérisation des conte- } \\
\text { ent apprécier l'incidence sur la } \\
\text { oduction et de la consommation } \\
\text { ée sur l'approche d'Andrew Stir- } \\
\text { ité culturelle est analysée selon } \\
\text { produite et consommée, l'équi- } \\
\text { ffférents titres et la disparité des } \\
\text { és. L'analyse révèle la baisse du } \\
\text { sétits et gros producteurs indé- } \\
\text { léments de réponse sur l'effet de } \\
\text { nypothèse de la longue traîne) et } \\
\text { rsité dans la part des ventes réa- }\end{array}$ \\
\hline
\end{tabular}

\section{RÉSUMÉ}

La question de la diversité culturelle est abordée sous l'angle de la mesure de la diversité du marché du film en salles et du film en vidéogrammes au cours des années 2000, sur la base d'un échantillon inédit de 5600 films diffusés en salles et de 6500 films enregistrés en vidéogrammes. La méthode retenue consiste, pour les deux marchés, à mesurer la diversité à partir des critères de variété, d'équilibre et de disparité. Des éléments d'appréciation de l'offre (films), de la distribution (copies) et de la demande (nombre de billets ou de vidéogrammes vendus) sont proposés pour six pays d'Europe : France, Danemark, Espagne, Pologne, Royaume-Uni, Suède. Le marché du film en vidéo se limite à la mesure du cas français. La méthode testée tend à montrer que la France est le pays où le marché du film en salles est le plus diversifié, mais que le marché du film en vidéo est beaucoup moins diversifié. Elle confirme les différences de diversité selon les circuits de commercialisation et, plus inédit, le niveau élevé de diversité du marché du film en vidéo sur l'internet.

\section{ABSTRACT}

The issue of cultural diversity is examined from the point of view of the diversity of the market for films shown in cinemas and films released as video recordings in the 2000s, based on a new sample of 5,600 films shown in cinemas, and 6,500 video film recordings. For both markets, the method used consisted of measuring diversity based on the criteria of variety, balance and range. The issues of increased supply (films), distribution (copies) and demand (number of tickets or video recordings sold) are covered for 6 European countries, namely: France, Denmark, Spain, Poland, the UK and Sweden. The video film market is covered for France only. The method used tends to indicate that France has the most diverse cinema film market, whilst its video film market is considerably less diverse. It confirms differences in diversity according to marketing channels whilst new research shows a higher level of diversity within the internet video film market.

Tous les documents publiés par le DEPS sont téléchargeables sur http://www.culture.gouv.fr/deps

Le DEPS n'assurant pas de diffusion physique de ses collections, nous vous proposons de vous informer régulièrement des parutions par message électronique. Pour ce faire, merci de bien vouloir nous communiquer votre courriel à l'adresse contact.deps@culture.gouv.fr 